\title{
Supplement Information
}

\section{One-year characterization of organic aerosol composition and sources using an extractive electrospray ionization time- of-flight mass spectrometer (EESI-TOF)}

Qi Lu ${ }^{1,2}$, Alexander L. Vogel ${ }^{1, a}$, Sepideh Esmaeilirad ${ }^{1}$, Liming $\mathrm{Cao}^{3}$, Jing Zheng ${ }^{4}$, Jean-Luc Jaffrezo ${ }^{5}$, Paola Fermo ${ }^{6}$, Anne KaspEr-Giebl ${ }^{7}$, Kaspar R. Daellenbach ${ }^{\mathrm{b}}$, Mindong $\mathrm{Chen}^{2}$, Xinlei $\mathrm{Ge}^{2}$, Urs Baltensperger ${ }^{1}$, André S. H. Prévôt ${ }^{1}$, Jay G. Slowik ${ }^{1}$

${ }^{1}$ Laboratory of Atmospheric Chemistry, Paul Scherrer Institute (PSI), 5232 Villigen, Switzerland

${ }^{2}$ Collavorative Innovation Center of Atmospheric Environment and Equipment Technology, Nanjing University of Information Science \& Technology, Nanjing, 210044, China

${ }^{3}$ Key Laboratory for Urban Habitat Environmental Science and Technology, School of Environment and Energy, Peking University Shenzhen Graduate School, Shenzhen, 518055, China

${ }^{4}$ State Key Joint Laboratory of Environmental Simulation and Pollution Control, College of Environmental Sciences and Engineering, Peking University, Beijing 100871, China

${ }^{5}$ University Grenoble Alpes, CNRS, IGE, 38000 Grenoble, France

${ }^{6}$ Department of Chemistry, University of Milan, 20133 Milan, Italy

${ }^{7}$ Institute of Chemical Technologies and Analytics, Vienna University of Technology, 1060 Vienna, Austria

${ }^{a}$ now at: Institute for Atmospheric and Environmental Sciences, Goethe University, Frankfurt am Main, Germany

bow at: Institute for Atmospheric and Earth System Research, University of Helsinki, Finland

Correspondence to: Jay. G. Slowik (jay.slowik@psi.ch) and Andre Prévôt (andre.prevot@psi.ch) 


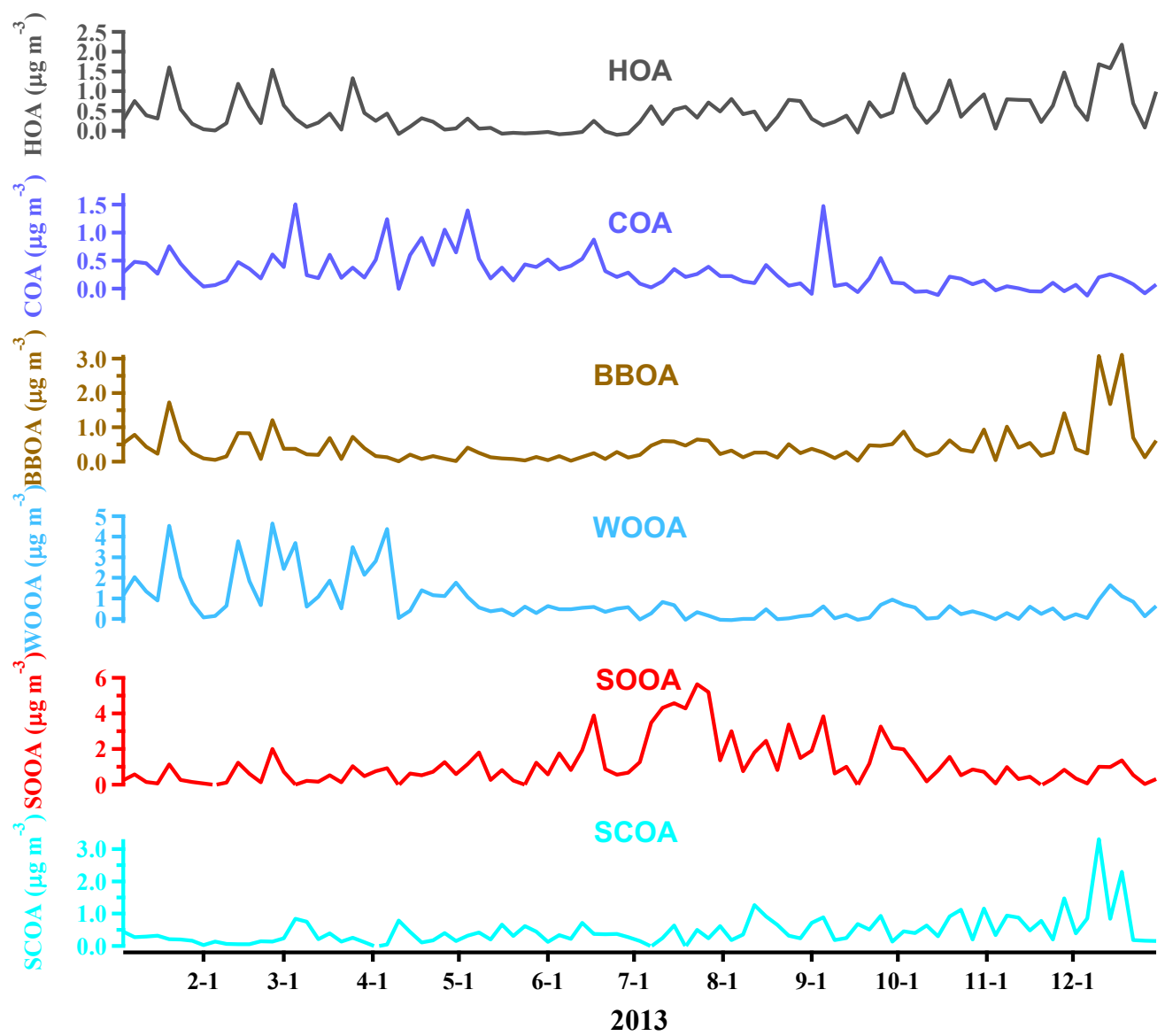

Fig. S1. Time series of AMS source apportionment factors. 


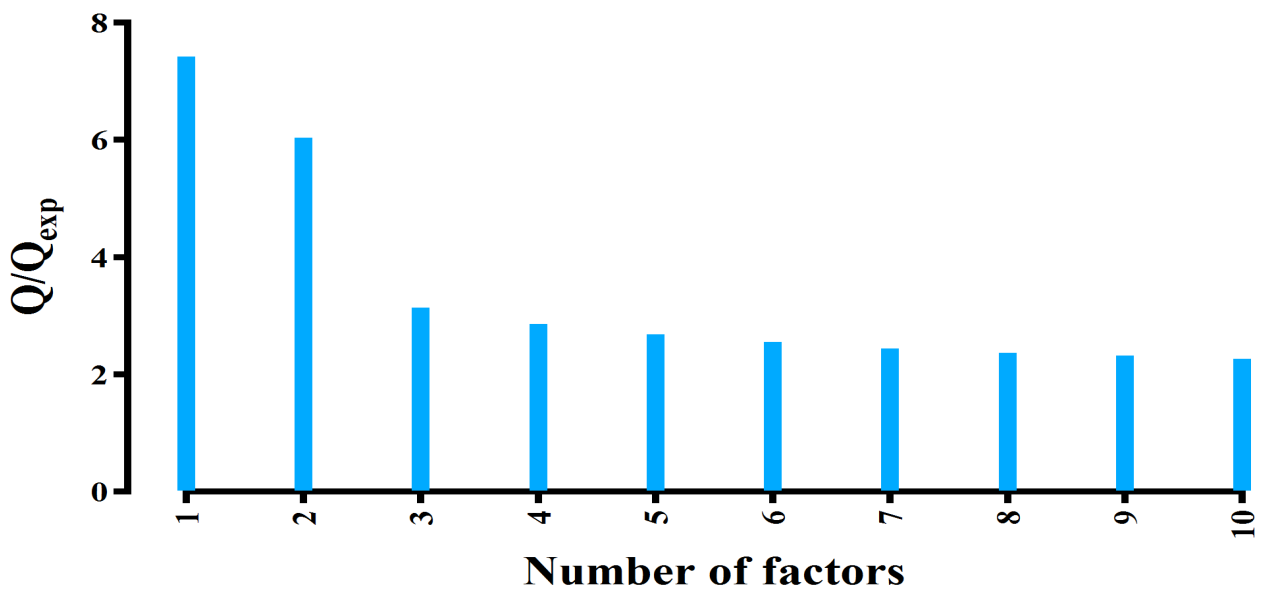

Fig. S2. $Q / Q_{\text {exp }}$ for the solutions consisting of 1 to 10 factors.

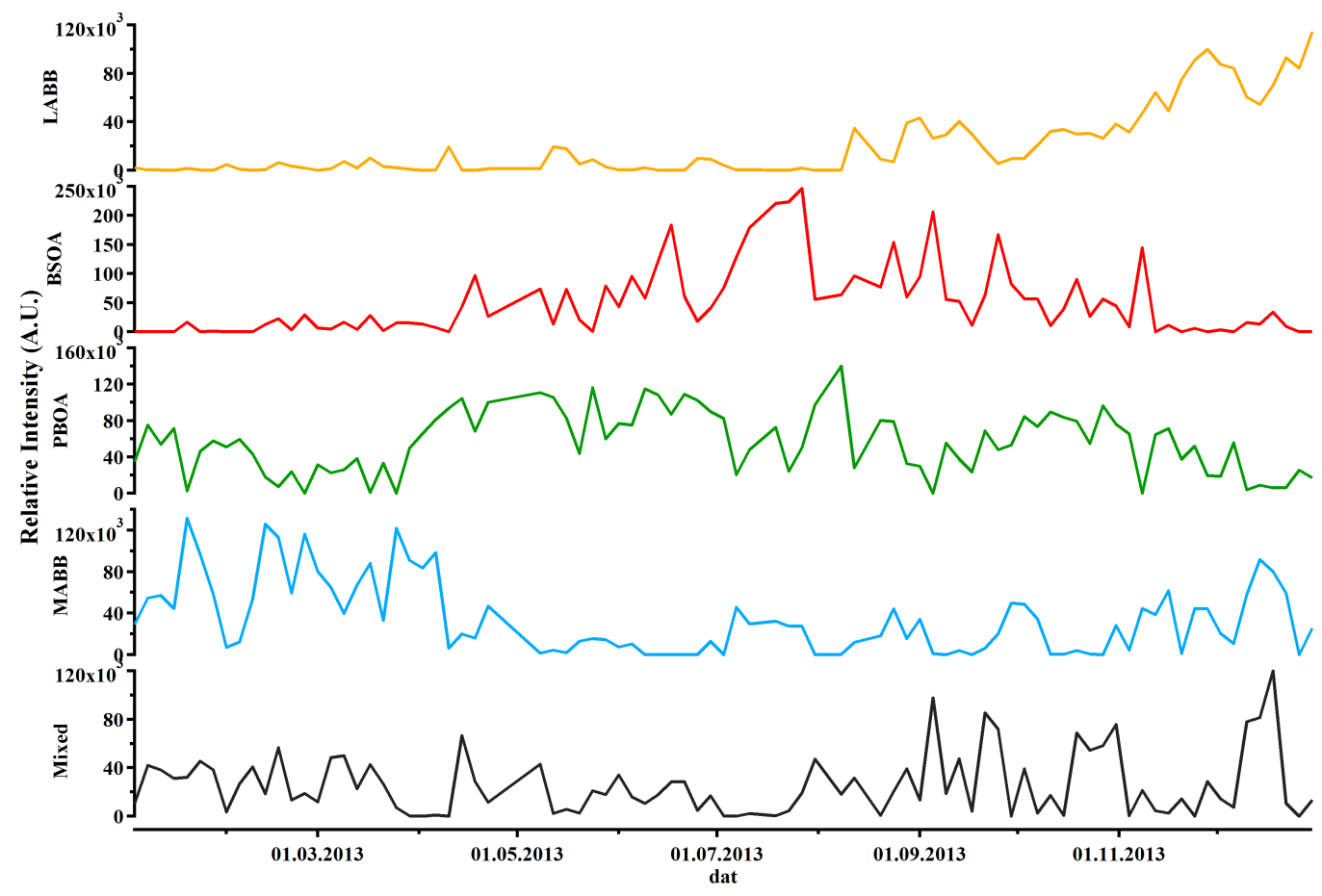




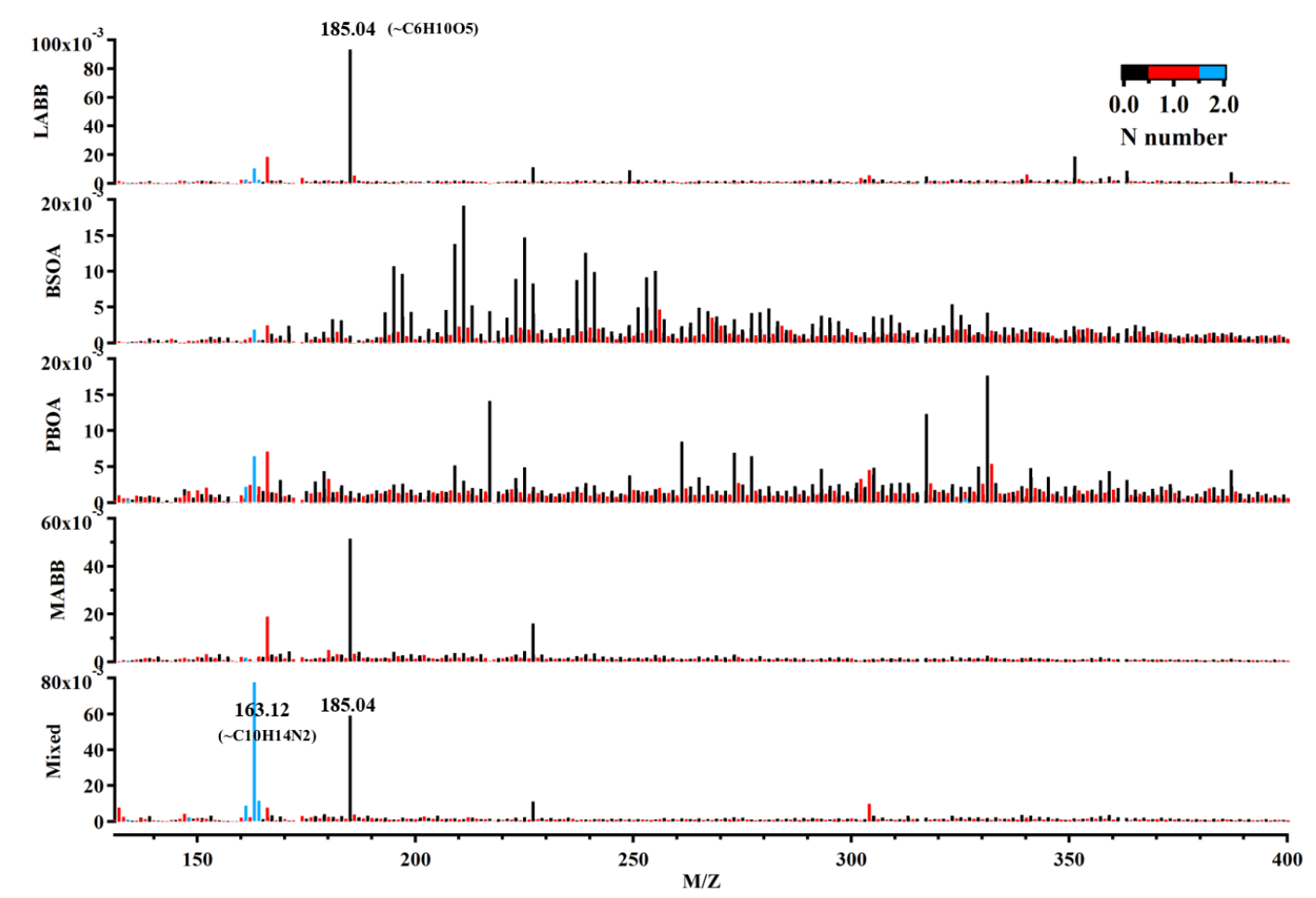

b)

Fig. S3. Time series (a) and profiles (b) of the EESI-TOF 5-factor PMF solution.

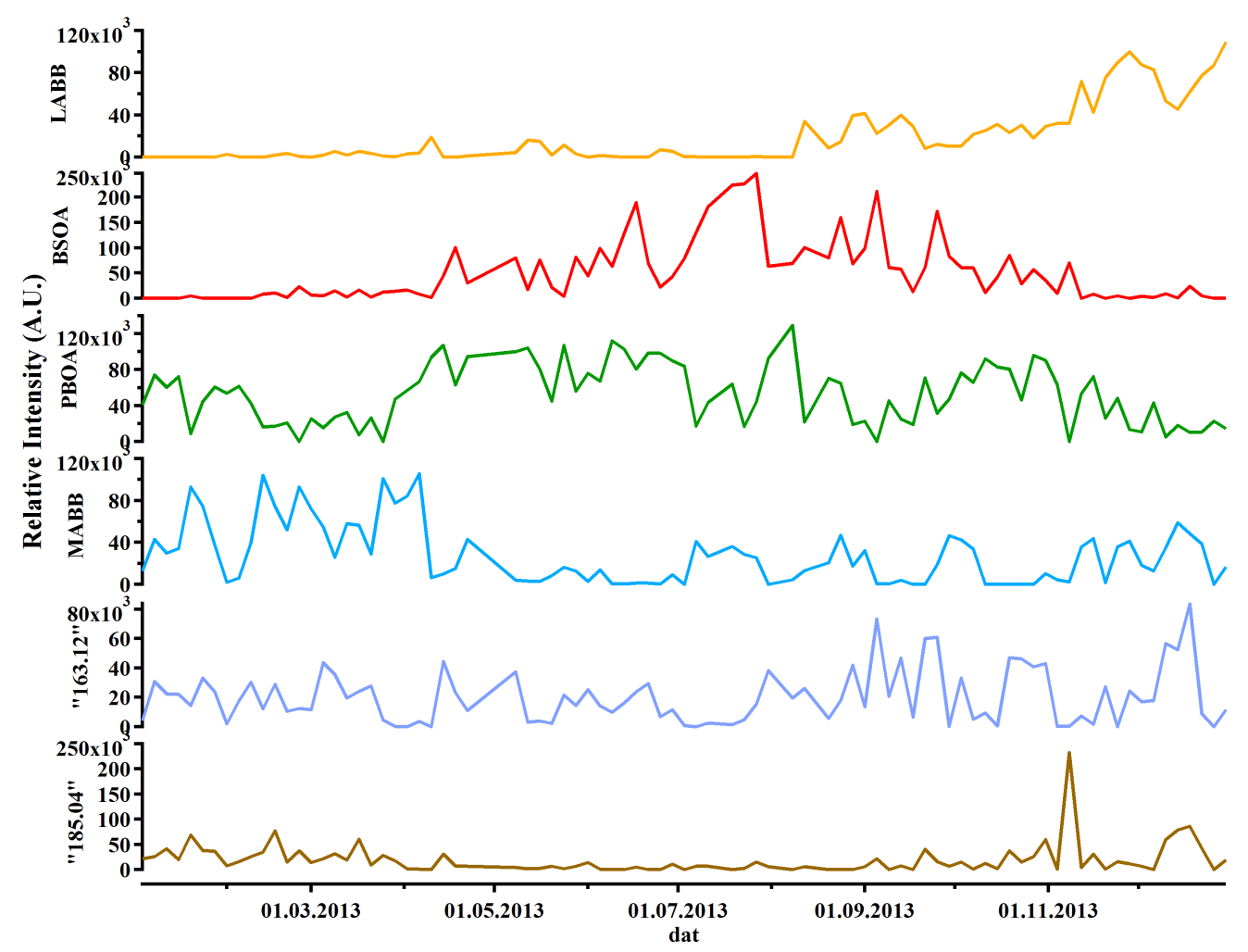



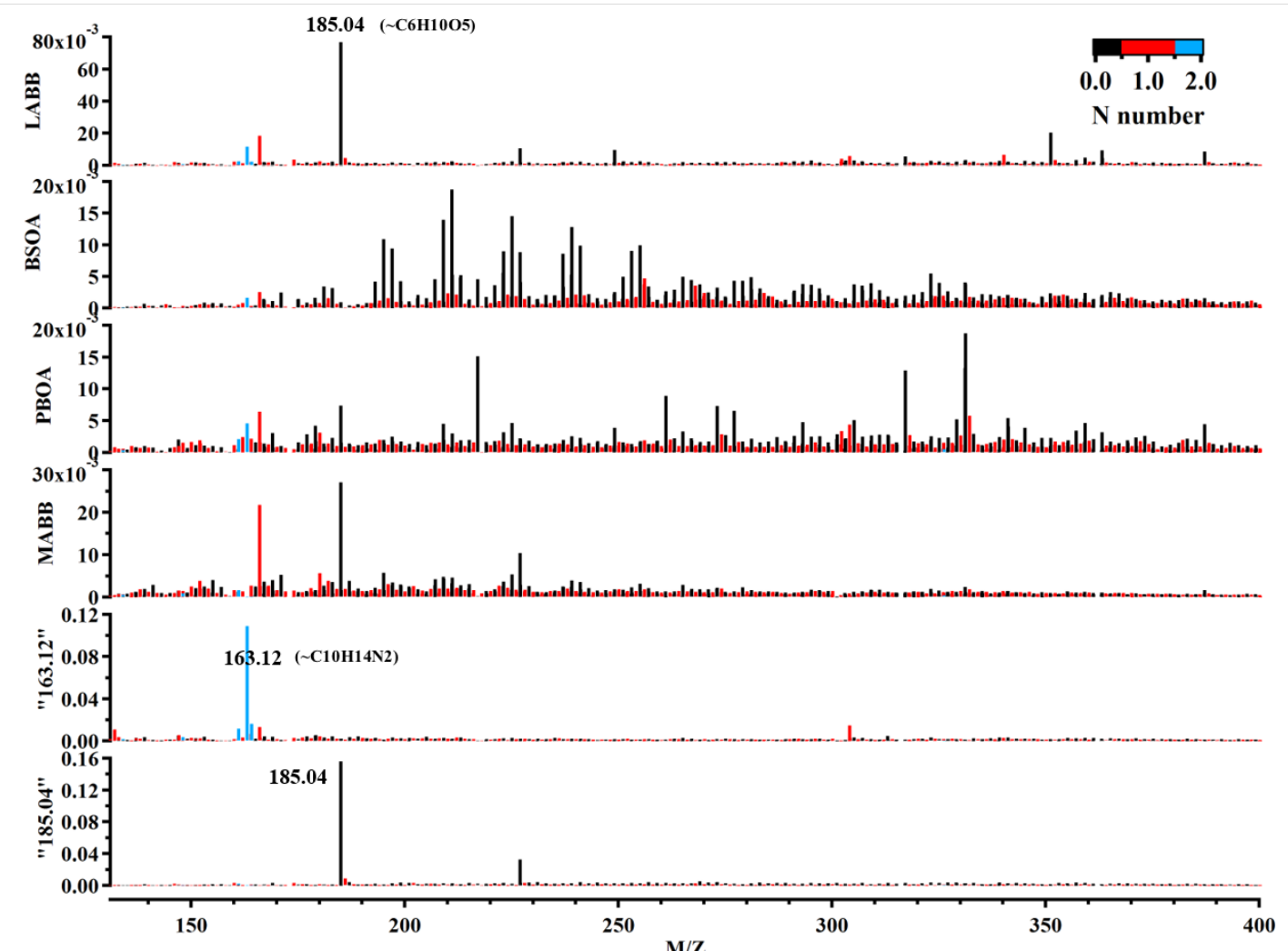

b)

Fig. S4. Time series (a) and profiles (b) of the EESI-TOF 6-factor PMF solution. 


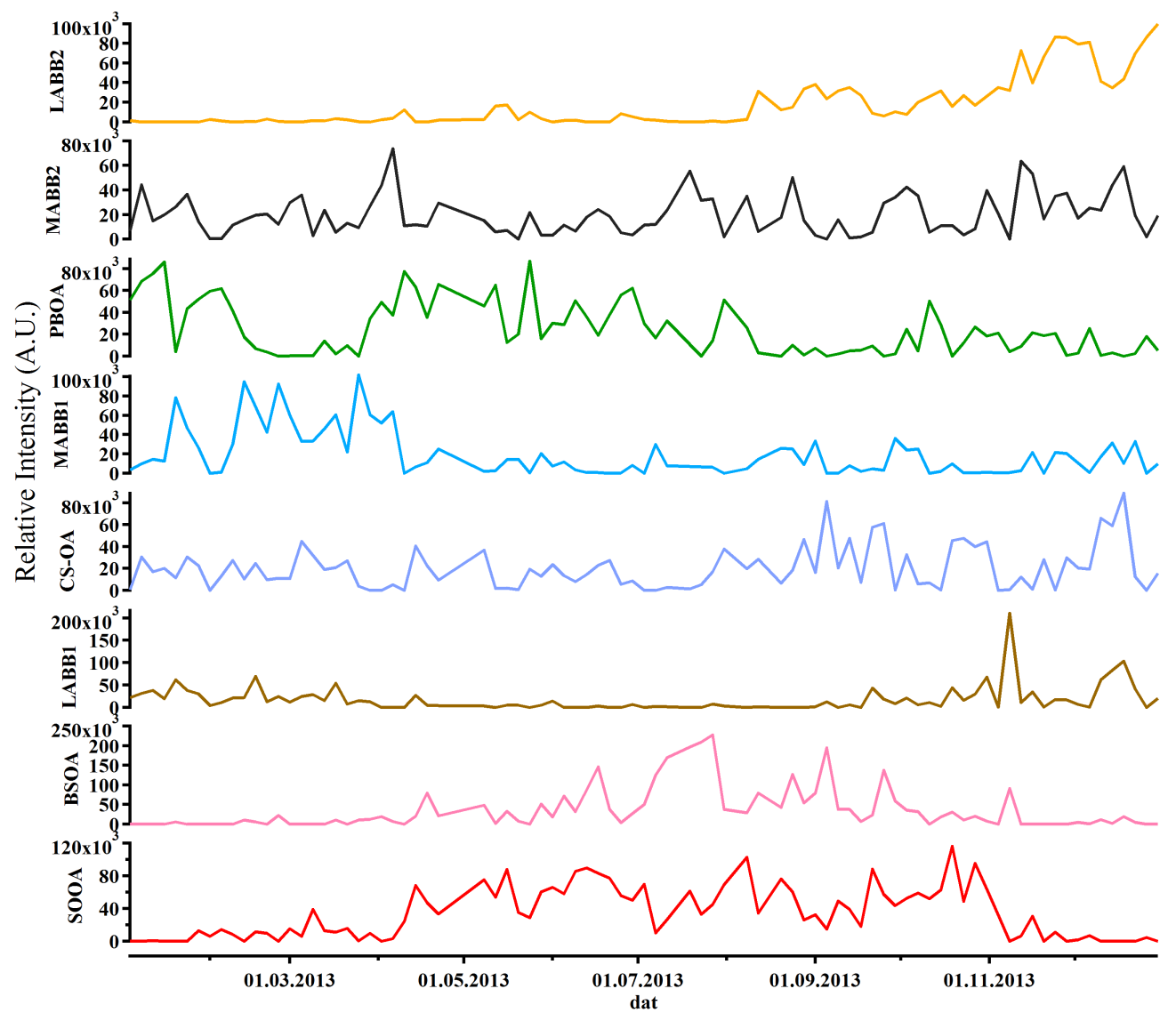

a)

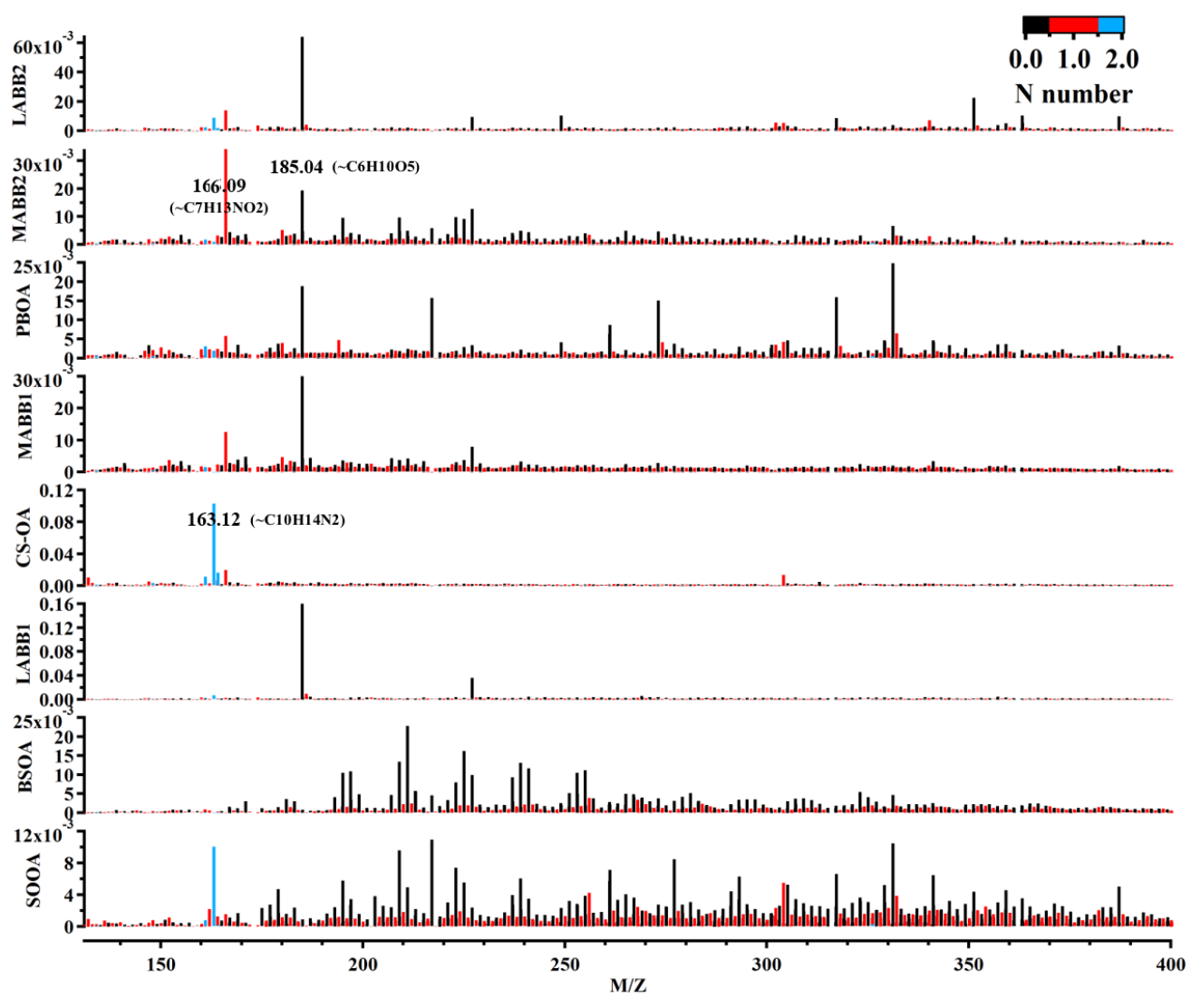


Fig. S5. Time series (a) and profiles (b) of EESI-TOF eight-factors solution.
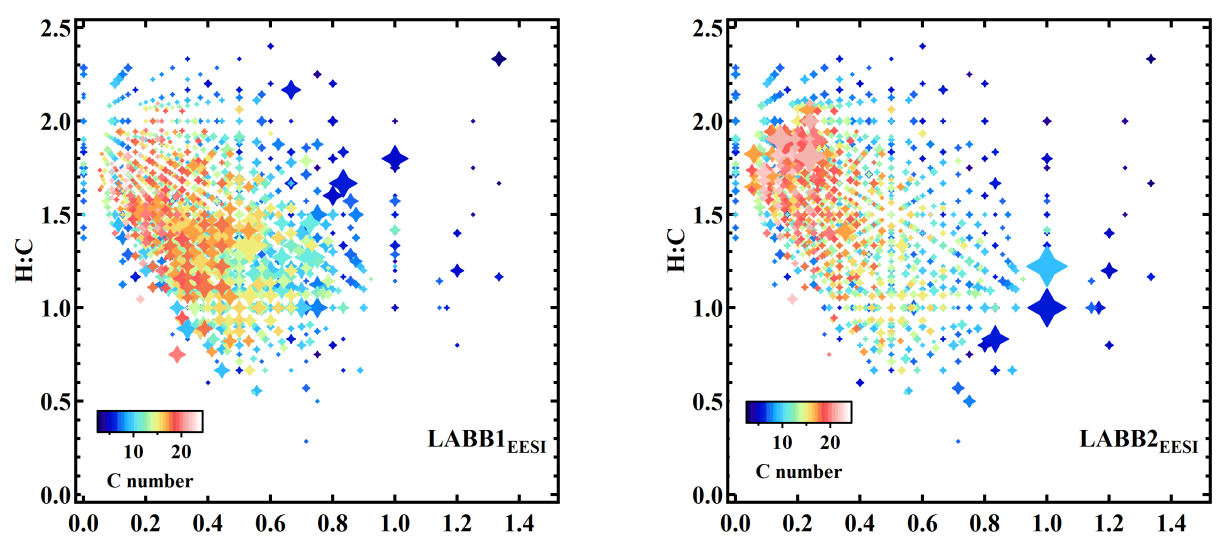

a)

b)
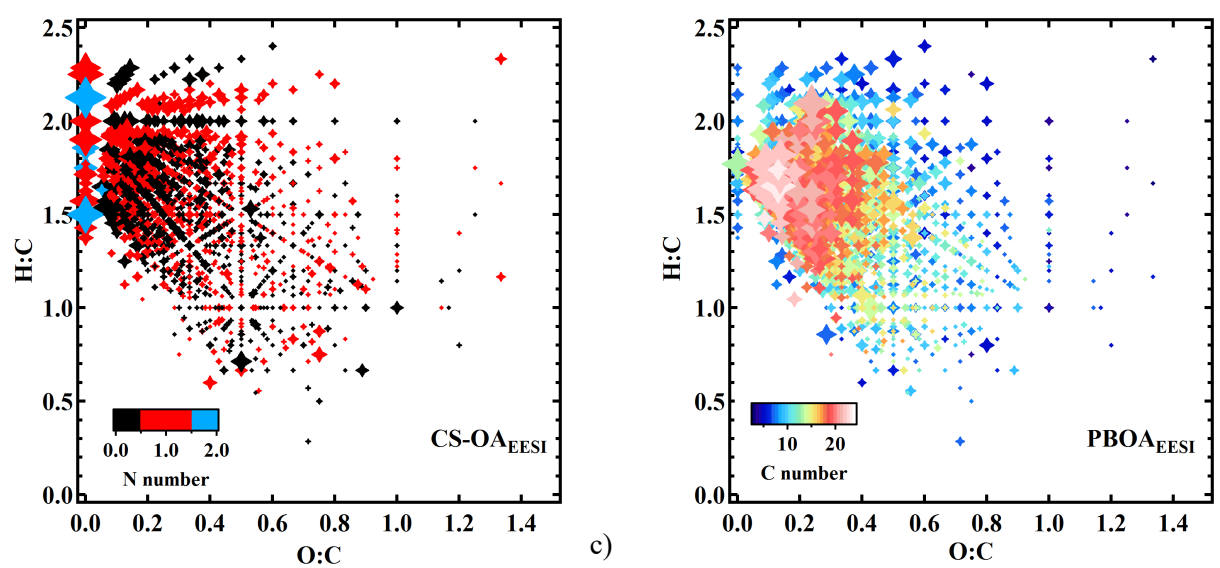

d)

Fig. S6. Van Krevelen plots (atomic H:C vs. O:C ratio) of the LABB1 $1_{\mathrm{EESI}}, \mathrm{LABB} 2_{\mathrm{EESI}}, \mathrm{CS}-\mathrm{OA} A_{\mathrm{EESI}}$ and $\mathrm{PBOA}_{\mathrm{EESI}}$ factor mass spectra. Points are sized by the fraction of each ion apportioned to LABB1 $1_{\mathrm{EESI}}$, $\mathrm{LABB}_{\mathrm{EESI}}, \mathrm{CS}-\mathrm{OA} \mathrm{A}_{\mathrm{EESI}}$ and $\mathrm{PBOA} \mathrm{AESI}_{\mathrm{E}}$, colored by number of carbon atoms and nitrogen number, respectively. 


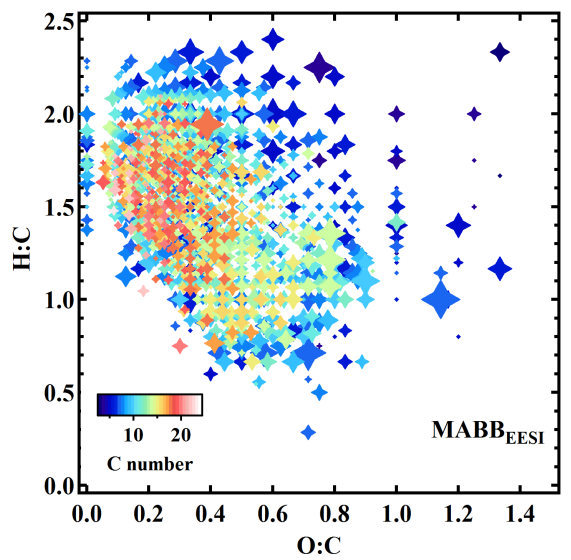

a)
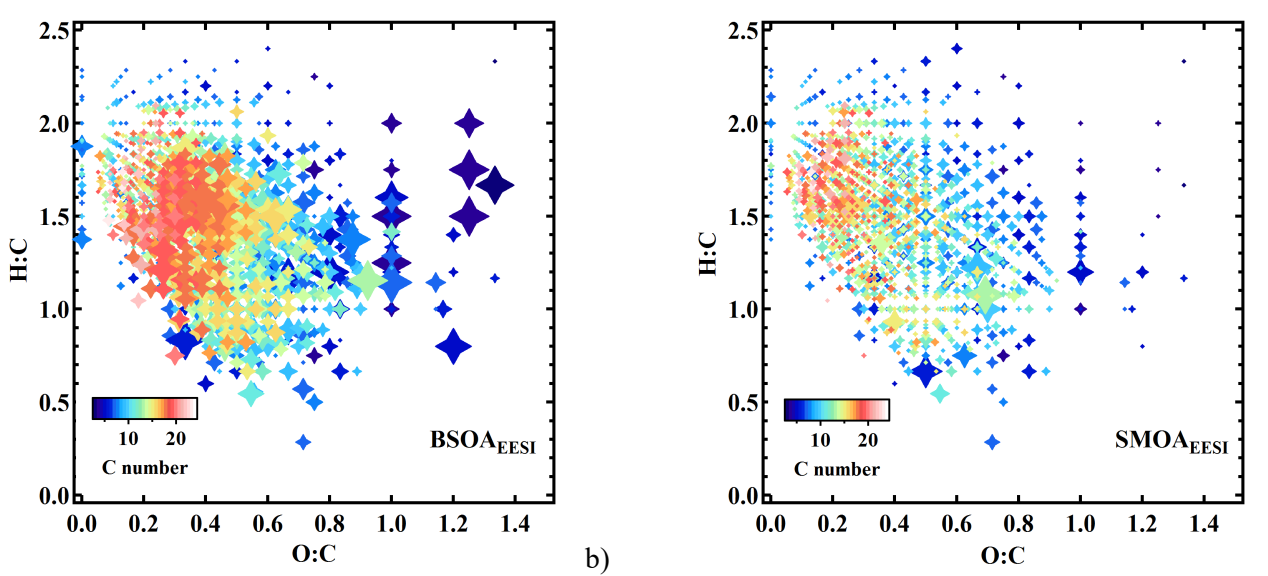

b)

c)

Fig. S7. Van Krevelen plot (atomic H:C vs. O:C ratio) of the MABB1 $1_{\mathrm{EESI}}, \mathrm{SMOA}_{\mathrm{EESI}}$ and BSOA $\mathrm{AESI}_{\mathrm{E}}$ factor mass spectra. Points are sized by the fraction of each ion apportioned to each factor and colored by number of carbon atoms and nitrogen number.
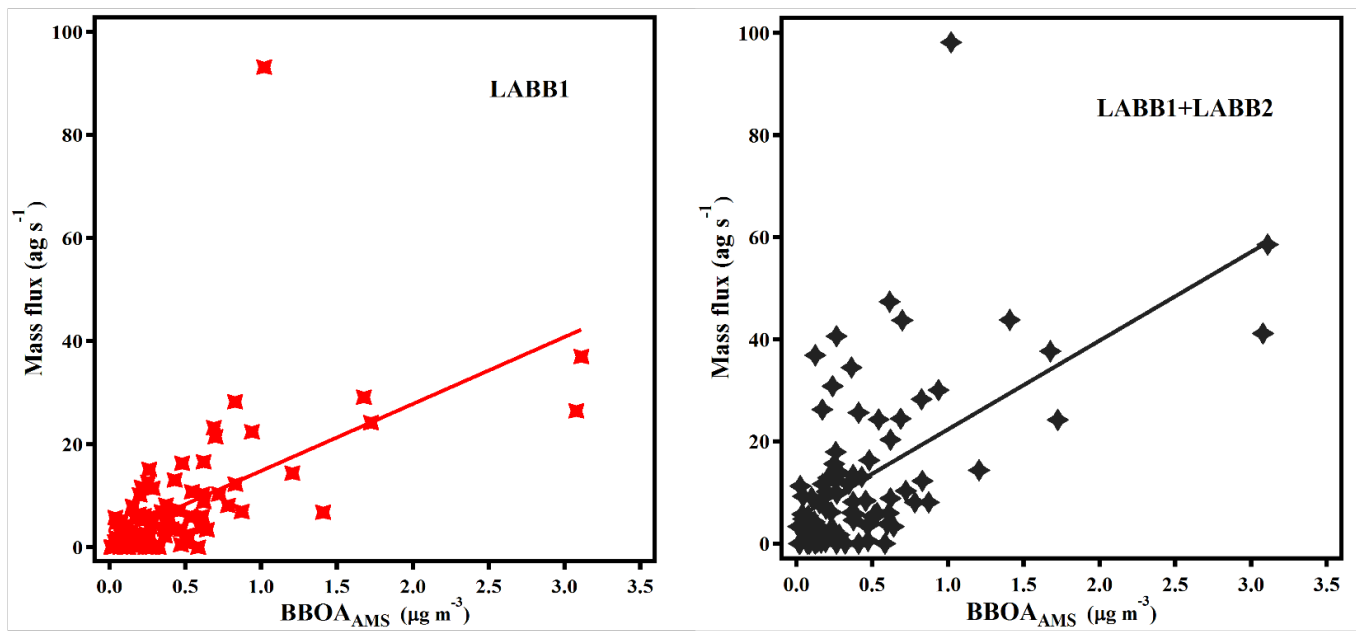

Fig. S8. Scatter plot of the LABB1 $1_{\mathrm{EESI}}$ factor and $\mathrm{LABB} 1_{\mathrm{EESI}}+\mathrm{LABB} 2_{\mathrm{EESI}}$ factor as a function of the 
$\mathrm{BBOA}_{\mathrm{AMS}}$ factor.

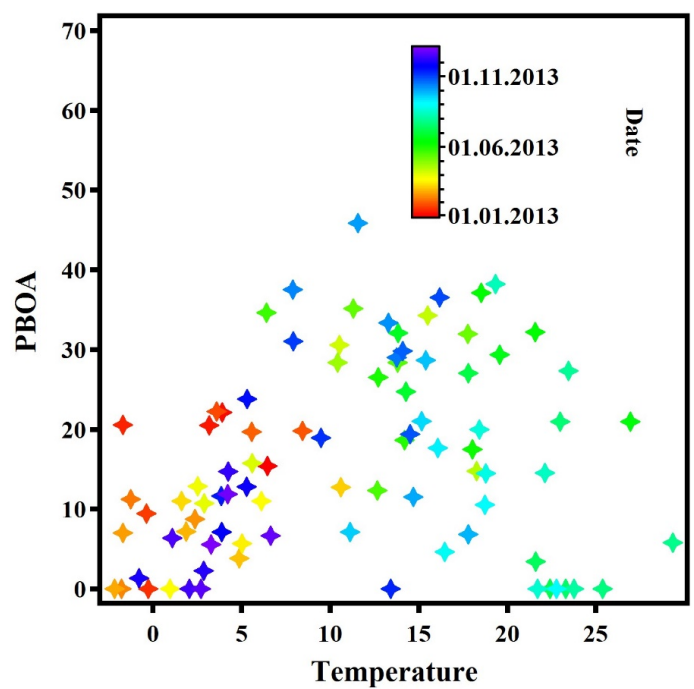

Fig. S9. The scatter plot of the temperature as a function of the PBOA factor of the EESI-TOF PMF.

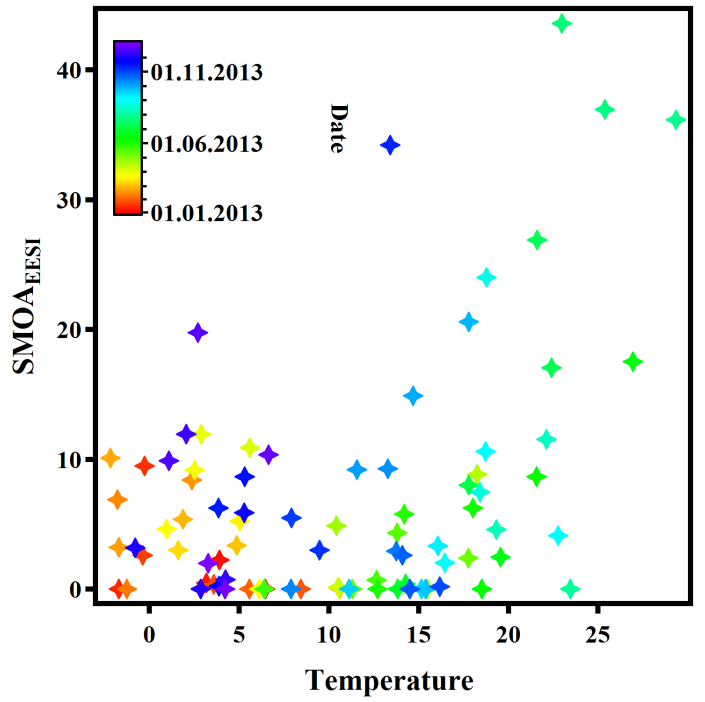

a) 


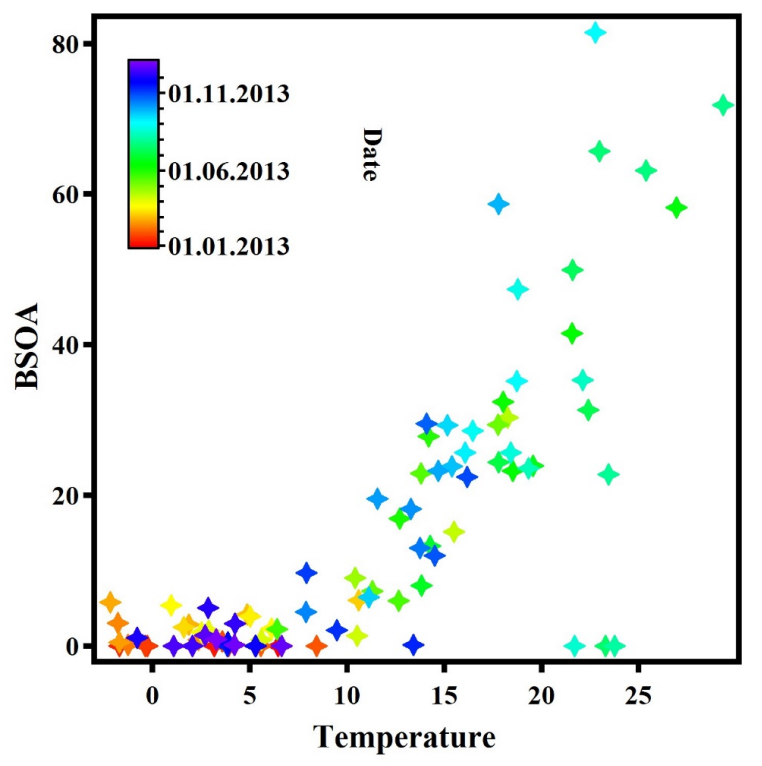

b)

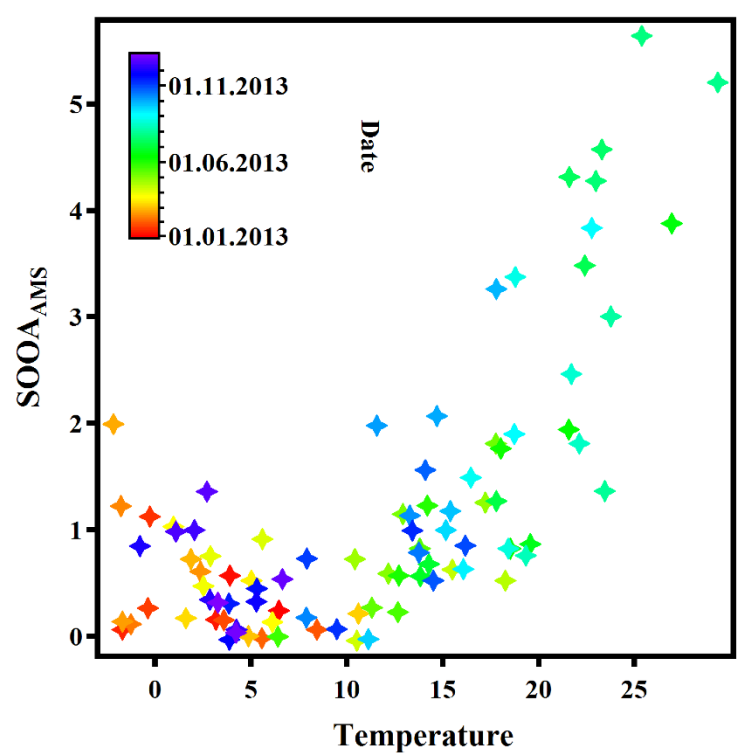

c)

Fig. S10. The scatter plot of the temperature as a function of the $\mathrm{SMOA}_{\mathrm{EESI}}$ factor (a), $\mathrm{BSOA}_{\mathrm{EESI}}$ factor (b) and $\mathrm{SOOA}_{\mathrm{AMS}}$ factor of the EESI-TOF PMF. 


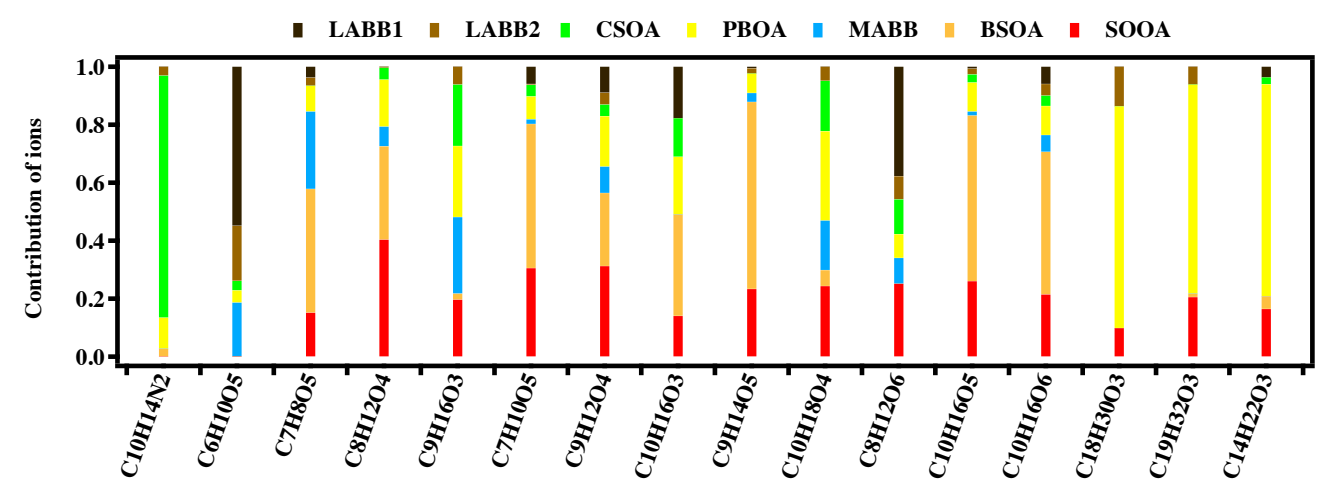

Fig. S11. Contribution of each ions to each factor from EESI-TOF PMF analysis.

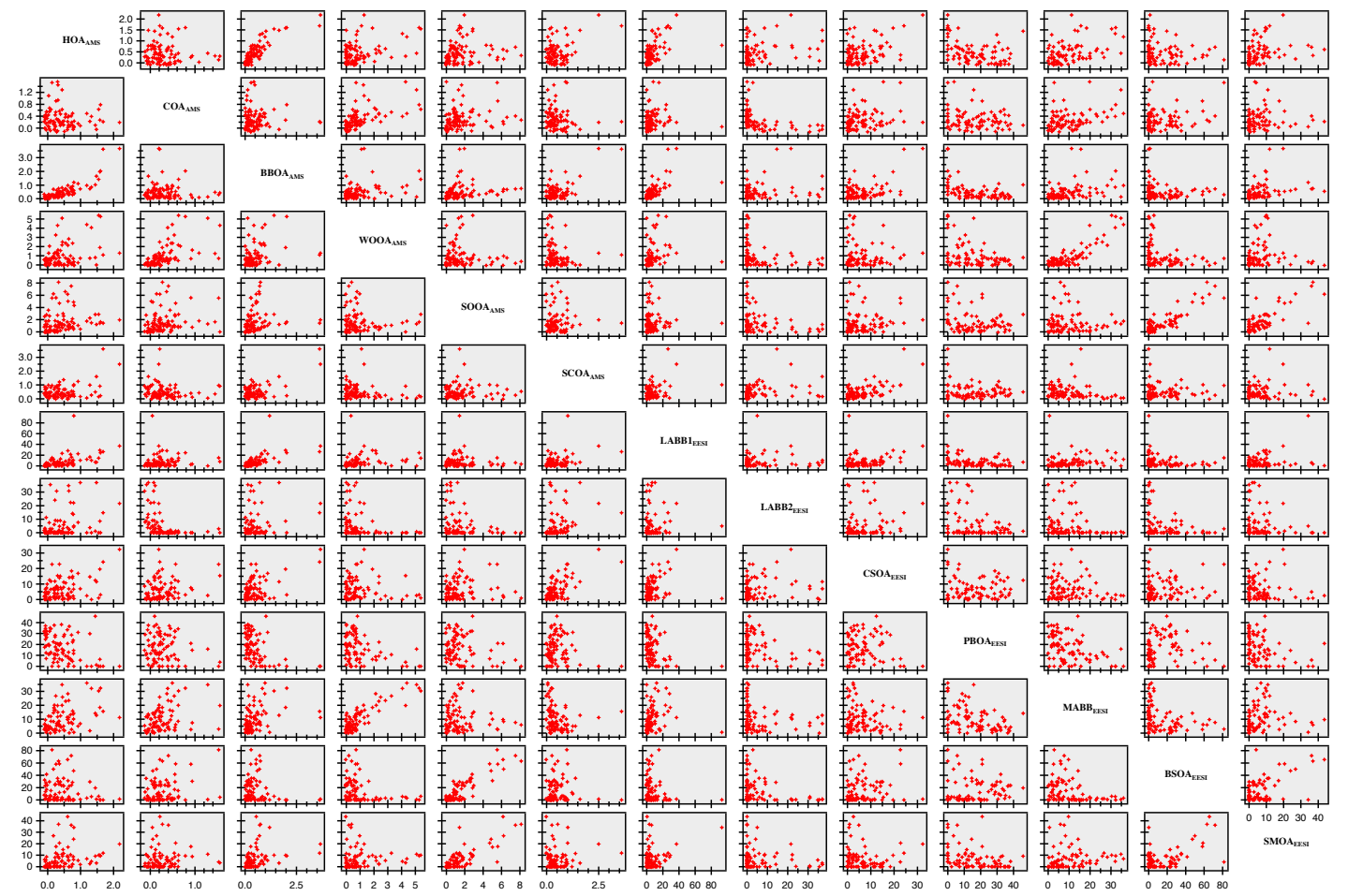

Fig. S12. Correlation between all the AMS and EESI factors.

Table S1. The list of the distinguished molecules of each factor.

\begin{tabular}{|l|l|r|}
\hline $\begin{array}{l}\text { Factor } \\
\text { name }\end{array}$ & Formula & \multicolumn{2}{l|}{$\mathrm{m} / \mathrm{z}$} \\
\hline LABB1 & C5H8O4 & 132.042 \\
\hline \multirow{2}{*}{} & C5H9NO5 & 163.048 \\
\cline { 2 - 3 }
\end{tabular}

\begin{tabular}{|l|r|} 
C6H10O5 & 162.053 \\
\hline C7H11NO6 & 205.059 \\
\hline C8H12O6 & 204.063 \\
\hline C8H15NO5 & 205.095 \\
\hline
\end{tabular}




\begin{tabular}{|c|c|}
\hline $\mathrm{C} 8 \mathrm{H} 7 \mathrm{NO} 3$ & 165.043 \\
\hline $\mathrm{C} 9 \mathrm{H} 11 \mathrm{NO} 3$ & 181.074 \\
\hline $\mathrm{C} 9 \mathrm{H} 13 \mathrm{NO} 3$ & 183.09 \\
\hline $\mathrm{C} 9 \mathrm{H} 8 \mathrm{O} 3$ & 164.047 \\
\hline $\mathrm{C} 10 \mathrm{H} 11 \mathrm{NO} 4$ & 209.069 \\
\hline $\mathrm{C} 10 \mathrm{H} 11 \mathrm{NO} 5$ & 225.064 \\
\hline C10H11NO6 & 241.059 \\
\hline $\mathrm{C} 10 \mathrm{H} 12 \mathrm{O} 4$ & 196.074 \\
\hline $\mathrm{C} 10 \mathrm{H} 12 \mathrm{O} 6$ & 228.063 \\
\hline $\mathrm{C} 10 \mathrm{H} 12 \mathrm{O} 7$ & 244.058 \\
\hline $\mathrm{C} 10 \mathrm{H} 13 \mathrm{NO} 5$ & 227.079 \\
\hline $\mathrm{C} 10 \mathrm{H} 1407$ & 246.074 \\
\hline $\mathrm{C} 11 \mathrm{H} 12 \mathrm{O} 4$ & 208.074 \\
\hline $\mathrm{C} 11 \mathrm{H} 12 \mathrm{O} 5$ & 224.069 \\
\hline C11H13NO4 & 223.085 \\
\hline C11H13NO5 & 239.079 \\
\hline C11H13NO6 & 255.074 \\
\hline $\mathrm{C} 11 \mathrm{H} 13 \mathrm{NO} 7$ & 271.069 \\
\hline $\mathrm{C} 11 \mathrm{H} 1404$ & 210.089 \\
\hline $\mathrm{C} 11 \mathrm{H} 14 \mathrm{O} 6$ & 242.079 \\
\hline $\mathrm{C} 11 \mathrm{H} 1407$ & 258.074 \\
\hline $\mathrm{C} 11 \mathrm{H} 1408$ & 274.069 \\
\hline C11H15NO4 & 225.1 \\
\hline $\mathrm{C} 11 \mathrm{H} 1607$ & 260.09 \\
\hline $\mathrm{C} 11 \mathrm{H} 1608$ & 276.085 \\
\hline $\mathrm{C} 12 \mathrm{H} 14 \mathrm{O} 3$ & 206.094 \\
\hline $\mathrm{C} 12 \mathrm{H} 14 \mathrm{O} 4$ & 222.089 \\
\hline $\mathrm{C} 12 \mathrm{H} 14 \mathrm{O} 7$ & 270.074 \\
\hline $\mathrm{C} 12 \mathrm{H} 15 \mathrm{NO} 4$ & 237.1 \\
\hline $\mathrm{C} 12 \mathrm{H} 15 \mathrm{NO} 5$ & 253.095 \\
\hline C12H15NO6 & 269.09 \\
\hline $\mathrm{C} 12 \mathrm{H} 16 \mathrm{O} 4$ & 224.105 \\
\hline
\end{tabular}

\begin{tabular}{|c|c|}
\hline $\mathrm{C} 12 \mathrm{H} 1607$ & 272.09 \\
\hline $\mathrm{C} 12 \mathrm{H} 1608$ & 288.085 \\
\hline $\mathrm{C} 13 \mathrm{H} 14 \mathrm{O} 5$ & 250.084 \\
\hline $\mathrm{C} 13 \mathrm{H} 14 \mathrm{O} 6$ & 266.079 \\
\hline $\mathrm{C} 13 \mathrm{H} 16 \mathrm{O} 4$ & 236.105 \\
\hline $\mathrm{C} 13 \mathrm{H} 16 \mathrm{O} 5$ & 252.1 \\
\hline $\mathrm{C} 13 \mathrm{H} 16 \mathrm{O} 6$ & 268.095 \\
\hline $\mathrm{C} 13 \mathrm{H} 17 \mathrm{NO} 4$ & 251.116 \\
\hline $\mathrm{C} 14 \mathrm{H} 14 \mathrm{O} 5$ & 262.084 \\
\hline $\mathrm{C} 14 \mathrm{H} 1605$ & 264.1 \\
\hline C14H17NO6 & 295.106 \\
\hline $\mathrm{C} 14 \mathrm{H} 18 \mathrm{O} 5$ & 266.115 \\
\hline $\mathrm{C} 14 \mathrm{H} 1807$ & 298.105 \\
\hline $\mathrm{C} 14 \mathrm{H} 2008$ & 316.116 \\
\hline $\mathrm{C} 15 \mathrm{H} 1606$ & 292.095 \\
\hline $\mathrm{C} 15 \mathrm{H} 1608$ & 324.085 \\
\hline $\mathrm{C} 15 \mathrm{H} 18 \mathrm{O} 5$ & 278.115 \\
\hline $\mathrm{C} 15 \mathrm{H} 18 \mathrm{O} 6$ & 294.11 \\
\hline $\mathrm{C} 15 \mathrm{H} 2006$ & 296.126 \\
\hline $\mathrm{C} 15 \mathrm{H} 2008$ & 328.116 \\
\hline $\mathrm{C} 15 \mathrm{H} 22 \mathrm{O} 8$ & 330.132 \\
\hline $\mathrm{C} 16 \mathrm{H} 18 \mathrm{O} 5$ & 290.115 \\
\hline $\mathrm{C} 16 \mathrm{H} 2005$ & 292.131 \\
\hline $\mathrm{C} 16 \mathrm{H} 2006$ & 308.126 \\
\hline $\mathrm{C} 16 \mathrm{H} 22 \mathrm{O} 10$ & 374.121 \\
\hline $\mathrm{C} 16 \mathrm{H} 22 \mathrm{O} 5$ & 294.147 \\
\hline $\mathrm{C} 16 \mathrm{H} 22 \mathrm{O} 6$ & 310.142 \\
\hline $\mathrm{C} 16 \mathrm{H} 22 \mathrm{O} 7$ & 326.137 \\
\hline C16H23NO5 & 309.158 \\
\hline $\mathrm{C} 16 \mathrm{H} 2409$ & 360.142 \\
\hline $\mathrm{C} 17 \mathrm{H} 2005$ & 304.131 \\
\hline $\mathrm{C} 17 \mathrm{H} 22 \mathrm{O} 5$ & 306.147 \\
\hline
\end{tabular}




\begin{tabular}{|c|c|c|c|c|c|}
\hline & $\mathrm{C} 17 \mathrm{H} 22 \mathrm{O} 6$ & 322.142 & & C17H33NO2 & 283.251 \\
\hline & $\mathrm{C} 17 \mathrm{H} 2408$ & 356.147 & & C17H35NO4 & 317.257 \\
\hline & $\mathrm{C} 17 \mathrm{H} 2409$ & 372.142 & & C18H31NO5 & 341.22 \\
\hline & $\mathrm{C} 18 \mathrm{H} 2007$ & 348.121 & & $\mathrm{C} 18 \mathrm{H} 32 \mathrm{O} 3$ & 296.235 \\
\hline & $\mathrm{C} 18 \mathrm{H} 22 \mathrm{O} 6$ & 334.142 & & $\mathrm{C} 18 \mathrm{H} 32 \mathrm{O} 5$ & 328.225 \\
\hline & $\mathrm{C} 18 \mathrm{H} 22 \mathrm{O} 8$ & 366.132 & & C18H33NO3 & 311.246 \\
\hline & $\mathrm{C} 18 \mathrm{H} 2405$ & 320.162 & & $\mathrm{C} 18 \mathrm{H} 33 \mathrm{NO} 4$ & 327.241 \\
\hline & $\mathrm{C} 18 \mathrm{H} 2407$ & 352.152 & & $\mathrm{C} 18 \mathrm{H} 3403$ & 298.251 \\
\hline & $\mathrm{C} 18 \mathrm{H} 24 \mathrm{O} 8$ & 368.147 & & $\mathrm{C} 18 \mathrm{H} 34 \mathrm{O} 5$ & 330.241 \\
\hline & $\mathrm{C} 19 \mathrm{H} 1806$ & 342.11 & & $\mathrm{C} 19 \mathrm{H} 32 \mathrm{O} 5$ & 340.225 \\
\hline & $\mathrm{C} 19 \mathrm{H} 22 \mathrm{O} 6$ & 346.142 & & C19H33NO5 & 355.236 \\
\hline & $\mathrm{C} 19 \mathrm{H} 22 \mathrm{O}$ & 362.137 & & С19H33NO6 & 371.231 \\
\hline & $\mathrm{C} 19 \mathrm{H} 2606$ & 350.173 & & $\mathrm{C} 19 \mathrm{H} 34 \mathrm{O} 4$ & 326.246 \\
\hline & $\mathrm{C} 2 \mathrm{OH} 24 \mathrm{O} 5$ & 344.162 & & $\mathrm{C} 19 \mathrm{H} 3603$ & 312.267 \\
\hline LABB2 & $\mathrm{C} 5 \mathrm{H} 6 \mathrm{O} 6$ & 162.016 & & $\mathrm{C} 19 \mathrm{H} 3604$ & 328.262 \\
\hline & $\mathrm{C} 6 \mathrm{H} 5 \mathrm{NO} 5$ & 171.017 & & $\mathrm{C} 2 \mathrm{OH} 29 \mathrm{NO} 4$ & 347.21 \\
\hline & $\mathrm{C} 6 \mathrm{H} 6 \mathrm{O} 6$ & 174.016 & & $\mathrm{C} 2 \mathrm{OH} 35 \mathrm{NO} 3$ & 337.262 \\
\hline & $\mathrm{C} 6 \mathrm{H} 6 \mathrm{O} 7$ & 190.011 & & $\mathrm{C} 2 \mathrm{OH} 38 \mathrm{O} 5$ & 358.272 \\
\hline & $\mathrm{C} 7 \mathrm{H} 4 \mathrm{O} 5$ & 168.006 & & $\mathrm{C} 21 \mathrm{H} 38 \mathrm{O} 3$ & 338.282 \\
\hline & $\mathrm{C} 8 \mathrm{H} 406$ & 196.001 & & $\mathrm{C} 21 \mathrm{H} 38 \mathrm{O} 5$ & 370.272 \\
\hline & $\mathrm{C} 13 \mathrm{H} 22 \mathrm{O} 3$ & 226.157 & & $\mathrm{C} 21 \mathrm{H} 40 \mathrm{O} 3$ & 340.298 \\
\hline & $\mathrm{C} 13 \mathrm{H} 24 \mathrm{O} 3$ & 228.173 & & $\mathrm{C} 21 \mathrm{H} 40 \mathrm{O} 4$ & 356.293 \\
\hline & $\mathrm{C} 15 \mathrm{H} 28 \mathrm{O} 4$ & 272.199 & & $\mathrm{C} 21 \mathrm{H} 42 \mathrm{O} 5$ & 374.303 \\
\hline & $\mathrm{C} 16 \mathrm{H} 25 \mathrm{NO} 3$ & 279.184 & & $\mathrm{C} 22 \mathrm{H} 37 \mathrm{NO} 2$ & 347.283 \\
\hline & $\mathrm{C} 16 \mathrm{H} 28 \mathrm{O} 3$ & 268.204 & & $\mathrm{C} 22 \mathrm{H} 38 \mathrm{O} 2$ & 334.287 \\
\hline & $\mathrm{C} 16 \mathrm{H} 29 \mathrm{NO} 5$ & 315.205 & & $\mathrm{C} 22 \mathrm{H} 40 \mathrm{O} 3$ & 352.298 \\
\hline & $\mathrm{C} 17 \mathrm{H} 28 \mathrm{O} 3$ & 280.204 & & $\mathrm{C} 24 \mathrm{H} 31 \mathrm{NO} 2$ & 365.236 \\
\hline & $\mathrm{C} 17 \mathrm{H} 29 \mathrm{NO} 2$ & 279.22 & CS-OA & $\mathrm{C} 6 \mathrm{H} 11 \mathrm{~N} 2$ & 111.092 \\
\hline & $\mathrm{C} 17 \mathrm{H} 3005$ & 314.209 & & $\mathrm{C} 6 \mathrm{H} 13 \mathrm{NO}$ & 115.1 \\
\hline & C17H31NO & 265.241 & & $\mathrm{C} 6 \mathrm{H} 13 \mathrm{NO} 3$ & 147.09 \\
\hline & $\mathrm{C} 17 \mathrm{H} 31 \mathrm{NO} 2$ & 281.236 & & C6H9NO & 111.068 \\
\hline & C17H31NO5 & 329.22 & & C7H10N & 108.081 \\
\hline
\end{tabular}




\begin{tabular}{|c|c|}
\hline C7H10O & 110.073 \\
\hline $\mathrm{C} 7 \mathrm{H} 11 \mathrm{~N}$ & 109.089 \\
\hline C7H11NO & 125.084 \\
\hline $\mathrm{C} 7 \mathrm{H} 12 \mathrm{~N}$ & 110.097 \\
\hline C7H12O & 112.089 \\
\hline $\mathrm{C} 7 \mathrm{H} 13 \mathrm{~N} 2$ & 125.108 \\
\hline C7H13NO & 127.1 \\
\hline $\mathrm{C} 7 \mathrm{H} 14 \mathrm{O}$ & 114.105 \\
\hline $\mathrm{C} 7 \mathrm{H} 14 \mathrm{O} 3$ & 146.094 \\
\hline $\mathrm{C} 7 \mathrm{H} 15 \mathrm{~N}$ & 113.121 \\
\hline C7H15NO & 129.115 \\
\hline C7H15NO2 & 145.11 \\
\hline C7H15NO3 & 161.105 \\
\hline C7H15NO4 & 177.1 \\
\hline $\mathrm{C} 7 \mathrm{H} 16 \mathrm{~N}$ & 114.128 \\
\hline $\mathrm{C} 7 \mathrm{H} 160$ & 116.12 \\
\hline $\mathrm{C} 8 \mathrm{H} 100$ & 122.073 \\
\hline $\mathrm{C} 8 \mathrm{H} 12 \mathrm{O}$ & 124.089 \\
\hline $\mathrm{C} 8 \mathrm{H} 13 \mathrm{~N}$ & 123.105 \\
\hline $\mathrm{C} 8 \mathrm{H} 14 \mathrm{~N}$ & 124.113 \\
\hline $\mathrm{C} 8 \mathrm{H} 14 \mathrm{~N} 2$ & 138.116 \\
\hline $\mathrm{C} 8 \mathrm{H} 140$ & 126.105 \\
\hline $\mathrm{C} 8 \mathrm{H} 14 \mathrm{O} 2$ & 142.099 \\
\hline $\mathrm{C} 8 \mathrm{H} 15 \mathrm{NO}$ & 141.115 \\
\hline $\mathrm{C} 8 \mathrm{H} 15 \mathrm{NO} 2$ & 157.11 \\
\hline $\mathrm{C} 8 \mathrm{H} 16$ & 112.125 \\
\hline $\mathrm{C} 8 \mathrm{H} 16 \mathrm{~N}$ & 126.128 \\
\hline $\mathrm{C} 8 \mathrm{H} 160$ & 128.12 \\
\hline $\mathrm{C} 8 \mathrm{H} 16 \mathrm{O} 2$ & 144.115 \\
\hline $\mathrm{C} 8 \mathrm{H} 16 \mathrm{O} 3$ & 160.11 \\
\hline $\mathrm{C} 8 \mathrm{H} 17 \mathrm{~N} 2$ & 141.139 \\
\hline $\mathrm{C} 8 \mathrm{H} 17 \mathrm{NO}$ & 143.131 \\
\hline
\end{tabular}

\begin{tabular}{|c|c|}
\hline $\mathrm{C} 8 \mathrm{H} 17 \mathrm{NO} 3$ & 175.121 \\
\hline $\mathrm{C} 8 \mathrm{H} 17 \mathrm{NO} 4$ & 191.116 \\
\hline $\mathrm{C} 8 \mathrm{H} 18 \mathrm{~N}$ & 128.144 \\
\hline $\mathrm{C} 8 \mathrm{H} 180$ & 130.136 \\
\hline $\mathrm{C} 8 \mathrm{H} 18 \mathrm{O} 3$ & 162.126 \\
\hline $\mathrm{C} 9 \mathrm{H} 15 \mathrm{~N}$ & 137.121 \\
\hline $\mathrm{C} 9 \mathrm{H} 15 \mathrm{NO}$ & 153.115 \\
\hline $\mathrm{C} 9 \mathrm{H} 17 \mathrm{NO}$ & 155.131 \\
\hline $\mathrm{C} 9 \mathrm{H} 18 \mathrm{O} 3$ & 174.126 \\
\hline $\mathrm{C} 9 \mathrm{H} 18 \mathrm{O} 4$ & 190.121 \\
\hline C9H19NO & 157.147 \\
\hline $\mathrm{C} 9 \mathrm{H} 19 \mathrm{NO} 3$ & 189.137 \\
\hline $\mathrm{C} 9 \mathrm{H} 20 \mathrm{O}$ & 144.152 \\
\hline $\mathrm{C} 9 \mathrm{H} 20 \mathrm{O} 2$ & 160.146 \\
\hline $\mathrm{C} 9 \mathrm{H} 20 \mathrm{O} 3$ & 176.141 \\
\hline $\mathrm{C} 10 \mathrm{H} 14 \mathrm{O} 3$ & 182.094 \\
\hline $\mathrm{C} 10 \mathrm{H} 15 \mathrm{~N} 2$ & 163.124 \\
\hline $\mathrm{C} 10 \mathrm{H} 15 \mathrm{NO}$ & 165.115 \\
\hline $\mathrm{C} 10 \mathrm{H} 17 \mathrm{NO}$ & 167.131 \\
\hline $\mathrm{C} 10 \mathrm{H} 180$ & 154.136 \\
\hline $\mathrm{C} 10 \mathrm{H} 19 \mathrm{~N}$ & 153.152 \\
\hline $\mathrm{C} 10 \mathrm{H} 19 \mathrm{NO}$ & 169.147 \\
\hline $\mathrm{C} 10 \mathrm{H} 19 \mathrm{NO} 2$ & 185.142 \\
\hline $\mathrm{C} 10 \mathrm{H} 200$ & 156.152 \\
\hline $\mathrm{C} 10 \mathrm{H} 21 \mathrm{NO}$ & 171.162 \\
\hline $\mathrm{C} 10 \mathrm{H} 21 \mathrm{NO} 3$ & 203.152 \\
\hline $\mathrm{C} 10 \mathrm{H} 21 \mathrm{NO} 4$ & 219.147 \\
\hline $\mathrm{C} 10 \mathrm{H} 22 \mathrm{O}$ & 158.167 \\
\hline $\mathrm{C} 11 \mathrm{H} 160$ & 164.12 \\
\hline $\mathrm{C} 11 \mathrm{H} 180$ & 166.136 \\
\hline $\mathrm{C} 11 \mathrm{H} 18 \mathrm{O} 2$ & 182.131 \\
\hline $\mathrm{C} 11 \mathrm{H} 1803$ & 198.126 \\
\hline
\end{tabular}




\begin{tabular}{|c|c|}
\hline $\mathrm{C} 11 \mathrm{H} 19 \mathrm{~N}$ & 165.152 \\
\hline C11H19NO & 181.147 \\
\hline $\mathrm{C} 11 \mathrm{H} 200$ & 168.152 \\
\hline $\mathrm{C} 11 \mathrm{H} 2003$ & 200.141 \\
\hline $\mathrm{C} 11 \mathrm{H} 21 \mathrm{~N}$ & 167.168 \\
\hline $\mathrm{C} 11 \mathrm{H} 21 \mathrm{NO}$ & 183.162 \\
\hline $\mathrm{C} 11 \mathrm{H} 21 \mathrm{NO} 2$ & 199.157 \\
\hline $\mathrm{C} 11 \mathrm{H} 22 \mathrm{O}$ & 170.167 \\
\hline $\mathrm{C} 11 \mathrm{H} 23 \mathrm{NO} 3$ & 217.168 \\
\hline $\mathrm{C} 11 \mathrm{H} 23 \mathrm{NO} 4$ & 233.163 \\
\hline $\mathrm{C} 12 \mathrm{H} 180$ & 178.136 \\
\hline $\mathrm{C} 12 \mathrm{H} 19 \mathrm{NO} 2$ & 209.142 \\
\hline $\mathrm{C} 12 \mathrm{H} 20 \mathrm{O} 2$ & 196.146 \\
\hline $\mathrm{C} 12 \mathrm{H} 2003$ & 212.141 \\
\hline $\mathrm{C} 12 \mathrm{H} 21 \mathrm{NO} 2$ & 211.157 \\
\hline $\mathrm{C} 12 \mathrm{H} 22 \mathrm{O}$ & 182.167 \\
\hline $\mathrm{C} 12 \mathrm{H} 23 \mathrm{NO}$ & 197.178 \\
\hline $\mathrm{C} 12 \mathrm{H} 23 \mathrm{NO} 2$ & 213.173 \\
\hline $\mathrm{C} 12 \mathrm{H} 25 \mathrm{NO}$ & 199.194 \\
\hline $\mathrm{C} 12 \mathrm{H} 25 \mathrm{NO} 4$ & 247.178 \\
\hline $\mathrm{C} 13 \mathrm{H} 20 \mathrm{O}$ & 192.152 \\
\hline $\mathrm{C} 13 \mathrm{H} 20 \mathrm{O} 2$ & 208.146 \\
\hline $\mathrm{C} 13 \mathrm{H} 21 \mathrm{NO}$ & 207.162 \\
\hline $\mathrm{C} 13 \mathrm{H} 21 \mathrm{NO} 2$ & 223.157 \\
\hline $\mathrm{C} 13 \mathrm{H} 22 \mathrm{O} 2$ & 210.162 \\
\hline $\mathrm{C} 13 \mathrm{H} 23 \mathrm{NO}$ & 209.178 \\
\hline $\mathrm{C} 13 \mathrm{H} 23 \mathrm{NO} 2$ & 225.173 \\
\hline $\mathrm{C} 13 \mathrm{H} 24 \mathrm{O}$ & 196.183 \\
\hline $\mathrm{C} 13 \mathrm{H} 25 \mathrm{NO}$ & 211.194 \\
\hline $\mathrm{C} 13 \mathrm{H} 26 \mathrm{O} 3$ & 230.188 \\
\hline $\mathrm{C} 13 \mathrm{H} 26 \mathrm{O} 4$ & 246.183 \\
\hline $\mathrm{C} 13 \mathrm{H} 27 \mathrm{NO} 3$ & 245.199 \\
\hline
\end{tabular}

\begin{tabular}{|c|c|c|}
\hline & $\mathrm{C} 14 \mathrm{H} 21 \mathrm{NO} 2$ & 235.157 \\
\hline & $\mathrm{C} 14 \mathrm{H} 22 \mathrm{O}$ & 206.167 \\
\hline & $\mathrm{C} 14 \mathrm{H} 22 \mathrm{O} 2$ & 222.162 \\
\hline & $\mathrm{C} 14 \mathrm{H} 23 \mathrm{NO} 2$ & 237.173 \\
\hline & $\mathrm{C} 14 \mathrm{H} 24 \mathrm{O} 3$ & 240.173 \\
\hline & $\mathrm{C} 14 \mathrm{H} 24 \mathrm{O} 3$ & 240.173 \\
\hline & $\mathrm{C} 14 \mathrm{H} 25 \mathrm{NO} 2$ & 239.189 \\
\hline & $\mathrm{C} 14 \mathrm{H} 27 \mathrm{NO} 2$ & 241.204 \\
\hline & $\mathrm{C} 14 \mathrm{H} 29 \mathrm{NO} 3$ & 259.215 \\
\hline & $\mathrm{C} 15 \mathrm{H} 22 \mathrm{O} 2$ & 234.162 \\
\hline & $\mathrm{C} 15 \mathrm{H} 23 \mathrm{NO} 4$ & 281.163 \\
\hline & $\mathrm{C} 15 \mathrm{H} 24 \mathrm{O} 2$ & 236.178 \\
\hline & $\mathrm{C} 15 \mathrm{H} 26 \mathrm{O} 3$ & 254.188 \\
\hline & $\mathrm{C} 15 \mathrm{H} 27 \mathrm{NO}$ & 237.209 \\
\hline & $\mathrm{C} 15 \mathrm{H} 27 \mathrm{NO} 2$ & 253.204 \\
\hline & $\mathrm{C} 15 \mathrm{H} 27 \mathrm{NO} 3$ & 269.199 \\
\hline & C15H27NO6 & 317.184 \\
\hline & $\mathrm{C} 15 \mathrm{H} 28 \mathrm{O} 3$ & 256.204 \\
\hline & $\mathrm{C} 15 \mathrm{H} 29 \mathrm{NO} 2$ & 255.22 \\
\hline & C15H29NO3 & 271.215 \\
\hline & $\mathrm{C} 15 \mathrm{H} 30 \mathrm{O} 3$ & 258.22 \\
\hline & $\mathrm{C} 16 \mathrm{H} 27 \mathrm{NO} 3$ & 281.199 \\
\hline & C16H31NO3 & 285.231 \\
\hline & $\mathrm{C} 17 \mathrm{H} 28 \mathrm{O} 2$ & 264.209 \\
\hline & $\mathrm{C} 17 \mathrm{H} 30 \mathrm{O}$ & 282.22 \\
\hline & $\mathrm{C} 18 \mathrm{H} 32 \mathrm{O} 4$ & 312.23 \\
\hline PBOA & $\mathrm{C9H} 16 \mathrm{O} 2$ & 156.115 \\
\hline & $\mathrm{C} 9 \mathrm{H} 17 \mathrm{NO} 2$ & 171.126 \\
\hline & $\mathrm{C} 12 \mathrm{H} 18 \mathrm{O} 2$ & 194.131 \\
\hline & $\mathrm{C} 12 \mathrm{H} 19 \mathrm{NO}$ & 193.147 \\
\hline & $\mathrm{C} 12 \mathrm{H} 22 \mathrm{O} 5$ & 246.147 \\
\hline & $\mathrm{C} 13 \mathrm{H} 1804$ & 238.121 \\
\hline
\end{tabular}




\begin{tabular}{|c|c|}
\hline $\mathrm{C} 13 \mathrm{H} 21 \mathrm{NO} 3$ & 239.152 \\
\hline $\mathrm{C} 13 \mathrm{H} 21 \mathrm{NO} 4$ & 255.147 \\
\hline $\mathrm{C} 13 \mathrm{H} 23 \mathrm{~N}$ & 193.183 \\
\hline $\mathrm{C} 14 \mathrm{H} 21 \mathrm{NO}$ & 251.152 \\
\hline $\mathrm{C} 14 \mathrm{H} 22 \mathrm{O} 3$ & 238.157 \\
\hline $\mathrm{C} 14 \mathrm{H} 22 \mathrm{O} 4$ & 254.152 \\
\hline $\mathrm{C} 14 \mathrm{H} 23 \mathrm{NO} 3$ & 253.168 \\
\hline $\mathrm{C} 14 \mathrm{H} 2406$ & 288.157 \\
\hline $\mathrm{C} 14 \mathrm{H} 2605$ & 274.178 \\
\hline $\mathrm{C} 14 \mathrm{H} 29 \mathrm{NO} 4$ & 275.21 \\
\hline $\mathrm{C} 15 \mathrm{H} 2004$ & 264.136 \\
\hline $\mathrm{C} 15 \mathrm{H} 22 \mathrm{O} 4$ & 266.152 \\
\hline $\mathrm{C} 15 \mathrm{H} 23 \mathrm{NO} 2$ & 249.173 \\
\hline $\mathrm{C} 15 \mathrm{H} 23 \mathrm{NO} 3$ & 265.168 \\
\hline $\mathrm{C} 15 \mathrm{H} 25 \mathrm{NO}$ & 235.194 \\
\hline $\mathrm{C} 15 \mathrm{H} 26 \mathrm{O}$ & 222.198 \\
\hline $\mathrm{C} 15 \mathrm{H} 2604$ & 270.183 \\
\hline $\mathrm{C} 16 \mathrm{H} 22 \mathrm{O} 3$ & 262.157 \\
\hline $\mathrm{C} 16 \mathrm{H} 24 \mathrm{O} 3$ & 264.173 \\
\hline $\mathrm{C} 16 \mathrm{H} 26 \mathrm{O} 4$ & 282.183 \\
\hline $\mathrm{C} 16 \mathrm{H} 27 \mathrm{NO}$ & 249.209 \\
\hline $\mathrm{C} 16 \mathrm{H} 3003$ & 270.22 \\
\hline C16H30O3 & 270.22 \\
\hline $\mathrm{C} 17 \mathrm{H} 22 \mathrm{O} 4$ & 290.152 \\
\hline $\mathrm{C} 17 \mathrm{H} 25 \mathrm{NO} 3$ & 291.184 \\
\hline $\mathrm{C} 17 \mathrm{H} 25 \mathrm{NO} 4$ & 307.178 \\
\hline $\mathrm{C} 17 \mathrm{H} 27 \mathrm{NO} 4$ & 309.194 \\
\hline $\mathrm{C} 18 \mathrm{H} 24 \mathrm{O} 4$ & 304.168 \\
\hline $\mathrm{C} 18 \mathrm{H} 26 \mathrm{O} 4$ & 306.183 \\
\hline $\mathrm{C} 18 \mathrm{H} 27 \mathrm{NO} 5$ & 337.189 \\
\hline $\mathrm{C} 18 \mathrm{H} 28 \mathrm{O} 4$ & 308.199 \\
\hline $\mathrm{C} 18 \mathrm{H} 29 \mathrm{NO} 2$ & 291.22 \\
\hline
\end{tabular}

\begin{tabular}{|c|c|c|}
\hline \multirow{2}{*}{\multicolumn{2}{|c|}{\begin{tabular}{|l|l|}
$\mathrm{C} 18 \mathrm{H} 30 \mathrm{O} 2$ \\
$\mathrm{C} 18 \mathrm{H} 30 \mathrm{O} 3$
\end{tabular}}} & 278.225 \\
\hline & & 294.22 \\
\hline & $\mathrm{C} 18 \mathrm{H} 30 \mathrm{O}$ & 310.215 \\
\hline & $\mathrm{C} 18 \mathrm{H} 32 \mathrm{O}$ & 360.215 \\
\hline & $\mathrm{C} 19 \mathrm{H} 2405$ & 332.162 \\
\hline & $\mathrm{C} 19 \mathrm{H} 28 \mathrm{O} 3$ & 304.204 \\
\hline & $\mathrm{C} 19 \mathrm{H} 28 \mathrm{O} 4$ & 320.199 \\
\hline & $\mathrm{C} 19 \mathrm{H} 3003$ & 306.22 \\
\hline & $\mathrm{C} 19 \mathrm{H} 30 \mathrm{O}$ & 322.215 \\
\hline & $\mathrm{C} 19 \mathrm{H} 31 \mathrm{NO} 2$ & 305.236 \\
\hline & $\mathrm{C} 19 \mathrm{H} 32 \mathrm{O}$ & 308.235 \\
\hline & $\mathrm{C} 19 \mathrm{H} 33 \mathrm{NO} 2$ & 307.251 \\
\hline & $\mathrm{C} 19 \mathrm{H} 3406$ & 358.236 \\
\hline & $\mathrm{C} 19 \mathrm{H} 3407$ & 374.231 \\
\hline & $\mathrm{C} 2 \mathrm{OH} 26 \mathrm{O} 5$ & 346.178 \\
\hline & $\mathrm{C} 2 \mathrm{OH} 30 \mathrm{O} 5$ & 350.209 \\
\hline & $\mathrm{C} 2 \mathrm{OH} 31 \mathrm{NO} 4$ & 349.225 \\
\hline & $\mathrm{C} 2 \mathrm{OH} 33 \mathrm{NO} 3$ & 335.246 \\
\hline & $\mathrm{C} 2 \mathrm{OH} 34 \mathrm{O} 6$ & 370.236 \\
\hline & $\mathrm{C} 20 \mathrm{H} 35 \mathrm{NO} 2$ & 321.267 \\
\hline & $\mathrm{C} 21 \mathrm{H} 3005$ & 362.209 \\
\hline & $\mathrm{C} 21 \mathrm{H} 34 \mathrm{O} 2$ & 318.256 \\
\hline & $\mathrm{C} 21 \mathrm{H} 34 \mathrm{O} 4$ & 350.246 \\
\hline & $\mathrm{C} 21 \mathrm{H} 4405$ & 376.319 \\
\hline & $\mathrm{C} 22 \mathrm{H} 34 \mathrm{O} 3$ & 346.251 \\
\hline & $\mathrm{C} 22 \mathrm{H} 36 \mathrm{O} 2$ & 332.272 \\
\hline & $\mathrm{C} 22 \mathrm{H} 37 \mathrm{NO} 3$ & 363.278 \\
\hline & $\mathrm{C} 22 \mathrm{H} 39 \mathrm{NO} 2$ & 349.298 \\
\hline & $\mathrm{C} 23 \mathrm{H} 38 \mathrm{O} 3$ & 362.282 \\
\hline MABB & C4H7NO4 & 133.038 \\
\hline & C4H9NO3 & 119.058 \\
\hline & $\mathrm{C} 5 \mathrm{H} 10 \mathrm{O} 3$ & 118.063 \\
\hline
\end{tabular}




\begin{tabular}{|c|c|}
\hline $\mathrm{C} 5 \mathrm{H} 11 \mathrm{NO} 3$ & 133.074 \\
\hline $\mathrm{C} 5 \mathrm{H} 11 \mathrm{NO} 4$ & 149.069 \\
\hline $\mathrm{C} 5 \mathrm{H} 12 \mathrm{O} 3$ & 120.079 \\
\hline C5H7NO6 & 177.027 \\
\hline $\mathrm{C} 5 \mathrm{H} 9 \mathrm{NO} 2$ & 115.063 \\
\hline C5H9NO3 & 131.058 \\
\hline $\mathrm{C} 5 \mathrm{H} 9 \mathrm{NO} 4$ & 147.053 \\
\hline $\mathrm{C} 6 \mathrm{H} 10 \mathrm{O} 2$ & 114.068 \\
\hline $\mathrm{C} 6 \mathrm{H} 10 \mathrm{O} 3$ & 130.063 \\
\hline $\mathrm{C} 6 \mathrm{H} 11 \mathrm{NO} 2$ & 129.079 \\
\hline C6H11NO3 & 145.074 \\
\hline $\mathrm{C} 6 \mathrm{H} 11 \mathrm{NO} 4$ & 161.069 \\
\hline C6H11NO5 & 177.064 \\
\hline $\mathrm{C} 6 \mathrm{H} 12 \mathrm{O} 2$ & 116.084 \\
\hline $\mathrm{C} 6 \mathrm{H} 12 \mathrm{O} 3$ & 132.079 \\
\hline $\mathrm{C} 6 \mathrm{H} 12 \mathrm{O} 4$ & 148.074 \\
\hline $\mathrm{C} 6 \mathrm{H} 13 \mathrm{NO} 2$ & 131.095 \\
\hline $\mathrm{C} 6 \mathrm{H} 14 \mathrm{O} 2$ & 118.099 \\
\hline $\mathrm{C} 6 \mathrm{H} 14 \mathrm{O} 3$ & 134.094 \\
\hline $\mathrm{C} 6 \mathrm{H} 5 \mathrm{NO} 4$ & 155.022 \\
\hline $\mathrm{C} 6 \mathrm{H} 6 \mathrm{O} 2$ & 110.037 \\
\hline $\mathrm{C} 6 \mathrm{H} 7 \mathrm{NO} 8$ & 221.017 \\
\hline $\mathrm{C} 6 \mathrm{H} 8 \mathrm{O} 2$ & 112.052 \\
\hline C6H9NO2 & 127.063 \\
\hline $\mathrm{C} 7 \mathrm{H} 10 \mathrm{O} 2$ & 126.068 \\
\hline C7H11NO2 & 141.079 \\
\hline $\mathrm{C} 7 \mathrm{H} 12 \mathrm{O} 2$ & 128.084 \\
\hline C7H13NO3 & 159.09 \\
\hline C7H13NO4 & 175.085 \\
\hline C7H13NO5 & 191.079 \\
\hline $\mathrm{C} 7 \mathrm{H} 14 \mathrm{O} 2$ & 130.099 \\
\hline $\mathrm{C} 7 \mathrm{H} 16 \mathrm{O} 2$ & 132.115 \\
\hline
\end{tabular}

\begin{tabular}{|c|c|}
\hline C7H16O3 & 148.11 \\
\hline $\mathrm{C} 7 \mathrm{H} 5 \mathrm{NO} 3$ & 151.027 \\
\hline $\mathrm{C} 7 \mathrm{H} 5 \mathrm{NO} 4$ & 167.022 \\
\hline C7H5NO5 & 183.017 \\
\hline C7H6O4 & 154.027 \\
\hline C7H7NO5 & 185.032 \\
\hline C7H7NO8 & 233.017 \\
\hline $\mathrm{C} 7 \mathrm{H} 806$ & 188.032 \\
\hline C7H9NO2 & 139.063 \\
\hline C7H9NO3 & 155.058 \\
\hline $\mathrm{C} 8 \mathrm{H} 10 \mathrm{O} 2$ & 138.068 \\
\hline $\mathrm{C} 8 \mathrm{H} 13 \mathrm{NO} 2$ & 155.095 \\
\hline $\mathrm{C} 8 \mathrm{H} 15 \mathrm{NO} 3$ & 173.105 \\
\hline $\mathrm{C} 8 \mathrm{H} 15 \mathrm{NO} 6$ & 221.09 \\
\hline $\mathrm{C} 8 \mathrm{H} 18 \mathrm{O} 2$ & 146.131 \\
\hline $\mathrm{C} 8 \mathrm{H} 6 \mathrm{O} 4$ & 166.027 \\
\hline C8H7NO6 & 213.027 \\
\hline $\mathrm{C} 8 \mathrm{H} 8 \mathrm{O} 4$ & 168.042 \\
\hline C8H9NO & 135.068 \\
\hline C8H9NO7 & 231.038 \\
\hline $\mathrm{C} 9 \mathrm{H} 1008$ & 246.038 \\
\hline $\mathrm{C} 9 \mathrm{H} 11 \mathrm{NO} 7$ & 245.054 \\
\hline $\mathrm{C} 9 \mathrm{H} 11 \mathrm{NO} 8$ & 261.048 \\
\hline $\mathrm{C} 9 \mathrm{H} 13 \mathrm{NO} 2$ & 167.095 \\
\hline $\mathrm{C} 9 \mathrm{H} 15 \mathrm{NO} 2$ & 169.11 \\
\hline $\mathrm{C} 9 \mathrm{H} 15 \mathrm{NO} 3$ & 185.105 \\
\hline $\mathrm{C} 9 \mathrm{H} 17 \mathrm{NO} 5$ & 219.111 \\
\hline $\mathrm{C} 9 \mathrm{H} 18 \mathrm{O} 2$ & 158.131 \\
\hline $\mathrm{C} 9 \mathrm{H} 19 \mathrm{NO} 5$ & 221.126 \\
\hline $\mathrm{C} 9 \mathrm{H} 606$ & 210.016 \\
\hline $\mathrm{C9H7NO4}$ & 193.038 \\
\hline C9H7NO5 & 209.032 \\
\hline
\end{tabular}




\begin{tabular}{|c|c|}
\hline $\mathrm{C} 9 \mathrm{H} 8 \mathrm{O}$ & 228.027 \\
\hline C9H9NO6 & 227.043 \\
\hline $\mathrm{C} 10 \mathrm{H} 1009$ & 274.032 \\
\hline $\mathrm{C} 10 \mathrm{H} 11 \mathrm{NO} 3$ & 193.074 \\
\hline $\mathrm{C} 10 \mathrm{H} 11 \mathrm{NO} 8$ & 273.048 \\
\hline C10H11NO9 & 289.043 \\
\hline $\mathrm{C} 10 \mathrm{H} 12 \mathrm{O} 2$ & 164.084 \\
\hline $\mathrm{C} 10 \mathrm{H} 12 \mathrm{O}$ & 276.048 \\
\hline $\mathrm{C} 10 \mathrm{H} 14 \mathrm{O} 2$ & 166.099 \\
\hline $\mathrm{C} 10 \mathrm{H} 15 \mathrm{NO} 2$ & 181.11 \\
\hline $\mathrm{C} 10 \mathrm{H} 17 \mathrm{NO} 2$ & 183.126 \\
\hline $\mathrm{C} 10 \mathrm{H} 17 \mathrm{NO} 3$ & 199.121 \\
\hline $\mathrm{C} 10 \mathrm{H} 17 \mathrm{NO} 4$ & 215.116 \\
\hline $\mathrm{C} 10 \mathrm{H} 19 \mathrm{NO} 4$ & 217.131 \\
\hline $\mathrm{C} 10 \mathrm{H} 19 \mathrm{NO} 5$ & 233.126 \\
\hline $\mathrm{C} 10 \mathrm{H} 2003$ & 188.141 \\
\hline C10H9NO6 & 239.043 \\
\hline C10H9NO7 & 255.038 \\
\hline C11H11NO7 & 269.054 \\
\hline $\mathrm{C} 11 \mathrm{H} 13 \mathrm{NO} 3$ & 207.09 \\
\hline C11H13NO8 & 287.064 \\
\hline $\mathrm{C} 11 \mathrm{H} 14 \mathrm{O} 2$ & 178.099 \\
\hline $\mathrm{C} 11 \mathrm{H} 16 \mathrm{O} 2$ & 180.115 \\
\hline $\mathrm{C} 11 \mathrm{H} 17 \mathrm{NO} 3$ & 211.121 \\
\hline C11H17NO4 & 227.116 \\
\hline C11H19NO4 & 229.131 \\
\hline $\mathrm{C} 11 \mathrm{H} 21 \mathrm{NO} 5$ & 247.142 \\
\hline C11H9NO7 & 267.038 \\
\hline C11H9NO8 & 283.033 \\
\hline $\mathrm{C} 12 \mathrm{H} 11 \mathrm{NO} 6$ & 265.059 \\
\hline $\mathrm{C} 12 \mathrm{H} 17 \mathrm{NO} 5$ & 255.111 \\
\hline $\mathrm{C} 12 \mathrm{H} 19 \mathrm{NO} 3$ & 225.137 \\
\hline
\end{tabular}

\begin{tabular}{|c|c|c|}
\hline & $\mathrm{C} 12 \mathrm{H} 19 \mathrm{NO} 4$ & 241.131 \\
\hline & $\mathrm{C} 12 \mathrm{H} 19 \mathrm{NO} 5$ & 257.126 \\
\hline & $\mathrm{C} 12 \mathrm{H} 21 \mathrm{NO} 4$ & 243.147 \\
\hline & $\mathrm{C} 12 \mathrm{H} 21 \mathrm{NO} 5$ & 259.142 \\
\hline & $\mathrm{C} 12 \mathrm{H} 21 \mathrm{NO} 6$ & 275.137 \\
\hline & $\mathrm{C} 13 \mathrm{H} 13 \mathrm{NO} 7$ & 295.069 \\
\hline & $\mathrm{C} 13 \mathrm{H} 19 \mathrm{NO} 3$ & 237.137 \\
\hline & C14H11NO9 & 337.043 \\
\hline & $\mathrm{C} 14 \mathrm{H} 15 \mathrm{NO} 10$ & 357.07 \\
\hline & C14H17NO11 & 375.08 \\
\hline & $\mathrm{C} 14 \mathrm{H} 19 \mathrm{NO} 10$ & 361.101 \\
\hline & C14H19NO11 & 377.096 \\
\hline & $\mathrm{C} 15 \mathrm{H} 15 \mathrm{NO} 10$ & 369.07 \\
\hline & $\mathrm{C} 15 \mathrm{H} 21 \mathrm{NO} 3$ & 263.152 \\
\hline BSOA & $\mathrm{C} 3 \mathrm{H} 5 \mathrm{NO} 4$ & 119.022 \\
\hline & C4H5NO4 & 131.022 \\
\hline & $\mathrm{C} 4 \mathrm{H} 6 \mathrm{O} 4$ & 118.027 \\
\hline & $\mathrm{C} 4 \mathrm{H} 6 \mathrm{O} 5$ & 134.022 \\
\hline & C4H7NO5 & 149.032 \\
\hline & $\mathrm{C} 5 \mathrm{H} 6 \mathrm{O} 3$ & 114.032 \\
\hline & $\mathrm{C} 5 \mathrm{H} 6 \mathrm{O} 4$ & 130.027 \\
\hline & $\mathrm{C} 5 \mathrm{H} 7 \mathrm{NO} 4$ & 145.038 \\
\hline & $\mathrm{C} 5 \mathrm{H} 8 \mathrm{O} 5$ & 148.037 \\
\hline & C6H5NO2 & 123.032 \\
\hline & C6H7NO4 & 157.038 \\
\hline & C7H10O6 & 190.048 \\
\hline & C7H11NO5 & 189.064 \\
\hline & C7H11NO7 & 221.054 \\
\hline & $\mathrm{C} 7 \mathrm{H} 807$ & 204.027 \\
\hline & $\mathrm{C} 8 \mathrm{H} 11 \mathrm{NO} 7$ & 233.054 \\
\hline & $\mathrm{C} 8 \mathrm{H} 12 \mathrm{O} 5$ & 188.069 \\
\hline & C8H9NO4 & 183.053 \\
\hline
\end{tabular}




\begin{tabular}{|c|c|}
\hline $\mathrm{C} 9 \mathrm{H} 12 \mathrm{O}$ & 216.063 \\
\hline $\mathrm{C} 9 \mathrm{H} 13 \mathrm{NO} 5$ & 215.079 \\
\hline $\mathrm{C} 9 \mathrm{H} 13 \mathrm{NO} 6$ & 231.074 \\
\hline $\mathrm{C} 9 \mathrm{H} 13 \mathrm{NO} 7$ & 247.069 \\
\hline $\mathrm{C} 9 \mathrm{H} 14 \mathrm{O} 5$ & 202.084 \\
\hline $\mathrm{C} 9 \mathrm{H} 8 \mathrm{O} 5$ & 196.037 \\
\hline $\mathrm{C} 9 \mathrm{H} 806$ & 212.032 \\
\hline $\mathrm{C} 10 \mathrm{H} 1005$ & 210.053 \\
\hline $\mathrm{C} 10 \mathrm{H} 1006$ & 226.048 \\
\hline $\mathrm{C} 10 \mathrm{H} 1406$ & 230.079 \\
\hline $\mathrm{C} 10 \mathrm{H} 15 \mathrm{NO} 7$ & 261.085 \\
\hline $\mathrm{C} 10 \mathrm{H} 1605$ & 216.1 \\
\hline $\mathrm{C} 10 \mathrm{H} 1606$ & 232.095 \\
\hline C11H17NO5 & 243.111 \\
\hline C11H17NO6 & 259.106 \\
\hline C11H17NO7 & 275.101 \\
\hline $\mathrm{C} 11 \mathrm{H} 1805$ & 230.115 \\
\hline C11H19NO7 & 277.116 \\
\hline C11H9NO5 & 235.048 \\
\hline $\mathrm{C} 12 \mathrm{H} 11 \mathrm{NO} 5$ & 249.064 \\
\hline $\mathrm{C} 12 \mathrm{H} 17 \mathrm{NO} 7$ & 287.101 \\
\hline $\mathrm{C} 12 \mathrm{H} 18 \mathrm{O} 5$ & 242.115 \\
\hline $\mathrm{C} 12 \mathrm{H} 1806$ & 258.11 \\
\hline C12H19NO6 & 273.121 \\
\hline $\mathrm{C} 12 \mathrm{H} 19 \mathrm{NO} 7$ & 289.116 \\
\hline $\mathrm{C} 12 \mathrm{H} 2005$ & 244.131 \\
\hline C13H13NO5 & 263.079 \\
\hline $\mathrm{C} 13 \mathrm{H} 15 \mathrm{NO} 5$ & 265.095 \\
\hline C13H19NO6 & 285.121 \\
\hline $\mathrm{C} 13 \mathrm{H} 2005$ & 256.131 \\
\hline $\mathrm{C} 13 \mathrm{H} 2007$ & 288.121 \\
\hline $\mathrm{C} 13 \mathrm{H} 22 \mathrm{O} 6$ & 274.142 \\
\hline
\end{tabular}

\begin{tabular}{|c|c|}
\hline $\mathrm{C} 14 \mathrm{H} 2006$ & 284.126 \\
\hline $\mathrm{C} 14 \mathrm{H} 22 \mathrm{O} 5$ & 270.147 \\
\hline $\mathrm{C} 14 \mathrm{H} 22 \mathrm{O} 6$ & 286.142 \\
\hline $\mathrm{C} 14 \mathrm{H} 23 \mathrm{NO} 4$ & 269.163 \\
\hline $\mathrm{C} 14 \mathrm{H} 24 \mathrm{O} 5$ & 272.162 \\
\hline $\mathrm{C} 14 \mathrm{H} 25 \mathrm{NO} 5$ & 287.173 \\
\hline $\mathrm{C} 15 \mathrm{H} 21 \mathrm{NO} 7$ & 327.132 \\
\hline $\mathrm{C} 15 \mathrm{H} 22 \mathrm{O} 6$ & 298.142 \\
\hline $\mathrm{C} 15 \mathrm{H} 22 \mathrm{O}$ & 314.137 \\
\hline $\mathrm{C} 15 \mathrm{H} 23 \mathrm{NO} 10$ & 377.132 \\
\hline $\mathrm{C} 15 \mathrm{H} 23 \mathrm{NO} 5$ & 297.158 \\
\hline $\mathrm{C} 15 \mathrm{H} 23 \mathrm{NO} 6$ & 313.153 \\
\hline $\mathrm{C} 15 \mathrm{H} 23 \mathrm{NO} 7$ & 329.148 \\
\hline C15H23NO9 & 361.137 \\
\hline $\mathrm{C} 15 \mathrm{H} 24 \mathrm{O} 5$ & 284.162 \\
\hline $\mathrm{C} 15 \mathrm{H} 24 \mathrm{O} 7$ & 316.152 \\
\hline $\mathrm{C} 15 \mathrm{H} 25 \mathrm{NO} 5$ & 299.173 \\
\hline $\mathrm{C} 15 \mathrm{H} 25 \mathrm{NO} 6$ & 315.168 \\
\hline $\mathrm{C} 15 \mathrm{H} 25 \mathrm{NO} 7$ & 331.163 \\
\hline $\mathrm{C} 15 \mathrm{H} 26 \mathrm{O} 5$ & 286.178 \\
\hline $\mathrm{C} 15 \mathrm{H} 28 \mathrm{O} 5$ & 288.194 \\
\hline $\mathrm{C} 16 \mathrm{H} 15 \mathrm{NO} 5$ & 301.095 \\
\hline C16H17NO5 & 303.111 \\
\hline C16H19NO5 & 305.126 \\
\hline $\mathrm{C} 16 \mathrm{H} 24 \mathrm{O} 10$ & 376.137 \\
\hline $\mathrm{C} 16 \mathrm{H} 24 \mathrm{O} 5$ & 296.162 \\
\hline $\mathrm{C} 16 \mathrm{H} 2406$ & 312.157 \\
\hline $\mathrm{C} 16 \mathrm{H} 24 \mathrm{O} 7$ & 328.152 \\
\hline $\mathrm{C} 16 \mathrm{H} 26 \mathrm{O} 5$ & 298.178 \\
\hline $\mathrm{C} 17 \mathrm{H} 24 \mathrm{O}$ & 340.152 \\
\hline $\mathrm{C} 17 \mathrm{H} 25 \mathrm{NO} 8$ & 371.158 \\
\hline $\mathrm{C} 17 \mathrm{H} 26 \mathrm{O} 5$ & 310.178 \\
\hline
\end{tabular}




\begin{tabular}{|c|c|c|}
\hline & C17H27NO5 & 325.189 \\
\hline & C17H27NO6 & 341.184 \\
\hline & $\mathrm{C} 17 \mathrm{H} 27 \mathrm{NO} 07$ & 357.179 \\
\hline & C17H29NO5 & 327.205 \\
\hline & $\mathrm{C} 18 \mathrm{H} 2004$ & 300.136 \\
\hline & $\mathrm{C} 18 \mathrm{H} 23 \mathrm{NO} 3$ & 301.168 \\
\hline & $\mathrm{C} 18 \mathrm{H} 26 \mathrm{O} 3$ & 290.188 \\
\hline & $\mathrm{C} 18 \mathrm{H} 2605$ & 322.178 \\
\hline & $\mathrm{C} 18 \mathrm{H} 27 \mathrm{NO} 7$ & 369.179 \\
\hline & $\mathrm{C} 18 \mathrm{H} 28 \mathrm{O} 5$ & 324.194 \\
\hline & $\mathrm{C} 18 \mathrm{H} 2807$ & 356.184 \\
\hline & $\mathrm{C} 18 \mathrm{H} 28 \mathrm{O} 8$ & 372.179 \\
\hline & $\mathrm{C} 18 \mathrm{H} 29 \mathrm{NO} 6$ & 355.2 \\
\hline & $\mathrm{C} 18 \mathrm{H} 29 \mathrm{NO} 7$ & 371.195 \\
\hline & $\mathrm{C} 18 \mathrm{H} 3005$ & 326.209 \\
\hline & $\mathrm{C} 19 \mathrm{H} 21 \mathrm{NO} 5$ & 343.142 \\
\hline & $\mathrm{C} 19 \mathrm{H} 23 \mathrm{NO} 5$ & 345.158 \\
\hline & $\mathrm{C} 19 \mathrm{H} 25 \mathrm{NO} 5$ & 347.173 \\
\hline & $\mathrm{C} 19 \mathrm{H} 2806$ & 352.189 \\
\hline & $\mathrm{C} 19 \mathrm{H} 2807$ & 368.184 \\
\hline & $\mathrm{C} 21 \mathrm{H} 2604$ & 342.183 \\
\hline SOOA & $\mathrm{C} 5 \mathrm{H} 7 \mathrm{NO} 3$ & 129.043 \\
\hline & $\mathrm{C} 6 \mathrm{H} 4 \mathrm{O} 3$ & 124.016 \\
\hline & $\mathrm{C} 6 \mathrm{H} 7 \mathrm{NO} 2$ & 125.048 \\
\hline & $\mathrm{C} 6 \mathrm{H} 8 \mathrm{O} 4$ & 144.042 \\
\hline & $\mathrm{C} 7 \mathrm{H} 10 \mathrm{O} 4$ & 158.058 \\
\hline & C7H10O5 & 174.053 \\
\hline & C7H11NO4 & 173.069 \\
\hline & $\mathrm{C} 7 \mathrm{H} 12 \mathrm{O} 4$ & 160.074 \\
\hline & $\mathrm{C} 7 \mathrm{H} 12 \mathrm{O} 5$ & 176.069 \\
\hline & $\mathrm{C} 7 \mathrm{H} 2 \mathrm{O} 5$ & 165.99 \\
\hline & $\mathrm{C} 7 \mathrm{H} 8 \mathrm{O} 4$ & 156.042 \\
\hline
\end{tabular}

\begin{tabular}{|c|c|}
\hline $\mathrm{C} 8 \mathrm{H} 1004$ & 170.058 \\
\hline $\mathrm{C} 8 \mathrm{H} 1005$ & 186.053 \\
\hline $\mathrm{C} 8 \mathrm{H} 12 \mathrm{O} 3$ & 156.079 \\
\hline $\mathrm{C} 8 \mathrm{H} 12 \mathrm{O} 4$ & 172.074 \\
\hline $\mathrm{C} 8 \mathrm{H} 13 \mathrm{NO} 4$ & 187.085 \\
\hline $\mathrm{C} 8 \mathrm{H} 13 \mathrm{NO} 6$ & 219.074 \\
\hline $\mathrm{C} 8 \mathrm{H} 14 \mathrm{O} 4$ & 174.089 \\
\hline $\mathrm{C} 8 \mathrm{H} 14 \mathrm{O} 5$ & 190.084 \\
\hline $\mathrm{C} 9 \mathrm{H} 11 \mathrm{NO} 5$ & 213.064 \\
\hline $\mathrm{C} 9 \mathrm{H} 12 \mathrm{O} 4$ & 184.074 \\
\hline $\mathrm{C} 9 \mathrm{H} 12 \mathrm{O} 5$ & 200.069 \\
\hline $\mathrm{C} 9 \mathrm{H} 14 \mathrm{O} 3$ & 170.094 \\
\hline $\mathrm{C} 9 \mathrm{H} 14 \mathrm{O} 4$ & 186.089 \\
\hline $\mathrm{C} 9 \mathrm{H} 14 \mathrm{O} 6$ & 218.079 \\
\hline $\mathrm{C} 9 \mathrm{H} 15 \mathrm{NO} 4$ & 201.1 \\
\hline $\mathrm{C9H} 15 \mathrm{NO} 6$ & 233.09 \\
\hline $\mathrm{C} 9 \mathrm{H} 16 \mathrm{O} 4$ & 188.105 \\
\hline $\mathrm{C} 9 \mathrm{H} 16 \mathrm{O} 5$ & 204.1 \\
\hline $\mathrm{C} 10 \mathrm{H} 14 \mathrm{O} 4$ & 198.089 \\
\hline $\mathrm{C} 10 \mathrm{H} 14 \mathrm{O} 5$ & 214.084 \\
\hline $\mathrm{C} 10 \mathrm{H} 16 \mathrm{O} 4$ & 200.105 \\
\hline C10H17NO6 & 247.106 \\
\hline $\mathrm{C} 10 \mathrm{H} 1805$ & 218.115 \\
\hline $\mathrm{C} 11 \mathrm{H} 1604$ & 212.105 \\
\hline $\mathrm{C} 11 \mathrm{H} 1605$ & 228.1 \\
\hline $\mathrm{C} 11 \mathrm{H} 1804$ & 214.121 \\
\hline $\mathrm{C} 12 \mathrm{H} 16 \mathrm{O} 3$ & 208.11 \\
\hline $\mathrm{C} 12 \mathrm{H} 20 \mathrm{O} 4$ & 228.136 \\
\hline $\mathrm{C} 13 \mathrm{H} 18 \mathrm{O} 3$ & 222.126 \\
\hline $\mathrm{C} 13 \mathrm{H} 20 \mathrm{O} 4$ & 240.136 \\
\hline $\mathrm{C} 14 \mathrm{H} 18 \mathrm{O} 3$ & 234.126 \\
\hline C14H19NO5 & 281.126 \\
\hline
\end{tabular}




\begin{tabular}{|l|l|}
$\mathrm{C} 14 \mathrm{H} 2003$ & 236.141 \\
\hline $\mathrm{C} 15 \mathrm{H} 2003$ & 248.141 \\
\hline $\mathrm{C} 15 \mathrm{H} 22 \mathrm{O} 3$ & 250.157 \\
\hline
\end{tabular}

\begin{tabular}{|l|l|r|}
$\mathrm{C} 16 \mathrm{H} 24 \mathrm{O} 2$ & 248.178 \\
\hline $\mathrm{C} 16 \mathrm{H} 25 \mathrm{NO} 4$ & 295.178 \\
\hline $\mathrm{C} 16 \mathrm{H} 26 \mathrm{O} 2$ & 250.193 \\
\hline
\end{tabular}

